\title{
Bleeding risk with dabigatran, rivaroxaban, warfarin, and antiplatelet agent in Asians with non-valvular atrial fibrillation
}

\author{
Yi-Hsin Chan ${ }^{1,2,3, *}$, Yung-Hsin Yeh ${ }^{1,2, *}$, Hui-Tzu Tu ${ }^{4}$, Chi-Tai Kuo ${ }^{1,2}$, Shang-Hung \\ Chang ${ }^{1,2,5}$, Lung-Sheng $\mathbf{W u}^{1,2}$, Hsin-Fu Lee ${ }^{1,2}$ and Lai-Chu See ${ }^{4,6,7}$ \\ ${ }^{1}$ The Cardiovascular Department, Chang-Gung Memorial Hospital, Taoyuan, Taiwan \\ ${ }^{2}$ College of Medicine, Chang-Gung University, Taoyuan, Taiwan \\ ${ }^{3}$ Microscopy Core Laboratory, Chang-Gung Memorial Hospital, Taoyuan, Taiwan \\ ${ }^{4}$ Department of Public Health, College of Medicine, Chang-Gung University, Taoyuan, Taiwan \\ ${ }^{5}$ Center for Big Data Analytics and Statistics, Chang-Gung Memorial Hospital, Taoyuan, Taiwan \\ ${ }^{6}$ Biostatistics Core Laboratory, Molecular Medicine Research Center, Chang-Gung University, Taoyuan, Taiwan \\ ${ }^{7}$ Division of Rheumatology, Allergy and Immunology, Department of Internal Medicine, Chang-Gung Memorial Hospital, \\ Taoyuan, Taiwan \\ ${ }^{*}$ These authors have contributed equally to this work \\ Correspondence to: Lai-Chu See, email: lichu@mail.cgu.edu.tw \\ Keywords: atrial fibrillation; direct thrombin inhibitor; factor xa inhibitor; hemorrhage; warfarin \\ Received: August 08, $2017 \quad$ Accepted: September 22, $2017 \quad$ Published: October 24, 2017 \\ Copyright: Chan et al. This is an open-access article distributed under the terms of the Creative Commons Attribution License 3.0 \\ (CC BY 3.0), which permits unrestricted use, distribution, and reproduction in any medium, provided the original author and source \\ are credited.
}

\section{ABSTRACT}

It is not understood if dabigatran or rivaroxaban are superior to antiplatelet agents (AA) for safety outcomes in Asians with non-valvular atrial fibrillation (NVAF). In this study we evaluated the bleeding risk of dabigatran, rivaroxaban, warfarin and AA in Asians with NVAF. This national retrospective cohort study analyzed 6,600, 3,167, 5,338 and 8,238 consecutive NVAF patients taking dabigatran, rivaroxaban, warfarin or AAs (including aspirin, clopidogrel or ticlopidine), respectively, from June 1, 2012 to December 31, 2013. Propensity-score weighting was used to balance covariates across study groups. Patients were followed until the first occurrence of any bleeding outcome or the end of the study. The $\mathrm{CHA}_{2} \mathrm{DS}_{2}$-VASc scores were 4.1 $\pm 1.6,4.1 \pm 1.6,3.3 \pm 1.8$ and $2.4 \pm 1.6$ for the dabigatran, rivaroxaban, warfarin, and AA groups, respectively. There were 5,822 (88.2\%) and $164(5.2 \%)$ patients taking low dose dabigatran and rivaroxaban, respectively. Hazard ratios ( $95 \%$ confidence intervals) for dabigatran, rivaroxaban, or warfarin versus AA were: intracranial hemorrhage, $0.36(0.23-0.57 ; P<0.0001), 0.25(0.10-0.64 ; P=0.0037)$ and 1.34 $(0.89-2.02 ; P=0.1664) ;$ gastrointestinal bleeding, $0.44(0.32-0.59 ; P<0.0001), 1.09(0.61-$ $1.93 ; P=0.7694)$, and $0.68(0.49-0.94 ; P=0.0189)$; and all hospitalized major bleeding, 0.41 $(0.32-0.53 ; P<0.0001), 0.65(0.41-1.03 ; P=0.0644)$ and $0.90(0.70-1.16 ; P=0.4130)$ after adjustment. The risk reduction of all major bleeding for dabigatran versus AA persisted on subgroup analysis. In conclusion, we observed that dabiagtran was associated with a lower risk of all major bleeding in Asians with NVAF, whereas rivaroxaban had a similar risk of all major bleeding compared with antiplatelet agents after adjustment of comorbidities.

\section{INTRODUCTION}

Although randomized trials have shown that warfarin significantly decreased the risk of thromboembolic events by $62 \%$ in patients with atrial fibrillation (AF), vitamin $\mathrm{K}$ antagonists (VKAs) remained underutilized in real world clinical practice. [1-3] There is a higher risk of intracranial hemorrhage (ICH) and other major bleeding events in Asians taking warfarin, compared to non-Asians. [4, 5] Data from a real-world 
registry study also showed that more than $65 \%$ of Asian patients on warfarin had a markedly low (16.7\%) time in therapeutic range (TTR). [6] Aspirin reduces the risk of stroke in AF patients by about $20 \%$ and is commonly used in AF patients for whom warfarin therapy is unsuitable. [1] Although aspirin is not as efficacious as warfarin in reducing the thromboembolic risk, it may be a more convenient choice than warfarin in certain patient populations, and may therefore be prescribed as an alternative to warfarin for stroke prevention especially in Asia.

The AVEROOSES trial reported that apixaban was non-inferior to aspirin for the risk of major bleeding (1.4\%/year versus 1.2\%/year, respectively) in $\mathrm{AF}$ patients. [7] These data suggested that apixaban is an attractive alternative to aspirin for stroke prevention in AF patients unsuitable for warfarin. However, there are no studies currently which directly compared the safety outcomes in AF patients treated with other nonvitamin $\mathrm{K}$ antagonist oral anticoagulants (NOACs), (e.g. dabigatran, edoxaban or rivaroxaban) versus those treated with aspirin. It is also unclear whether dabigatran or rivaroxaban is superior to antiplatelet agents (AA) (including aspirin, clopidogrel, or ticlopidine) for safety outcomes, specifically in Asians with AF. Therefore, in this study we evaluated the bleeding risk associated with dabigatran, rivaroxaban, warfarin compared to AA therapy in Asians with non-valvular AF.

\section{RESULTS}

\section{Participants}

Patient demographics and medication use before and after propensity score weighting are described in Tables 1 to 3 . This study enrolled a total of $6,600,3,167,5,338$ and 8,238 consecutive patients taking dabigatran, rivaroxaban, warfarin, and AA respectively, from June, 2012 to December, 2013. Analysis of the AA group showed that a total of 7,181 (87.2\%), $470(5.7 \%), 252(3.1 \%)$, and 335 (4.1\%) patients took aspirin, clopidogrel, ticlopidine, and dual AAs (mostly aspirin plus clopidogrel, $n=319,3.9 \%$ ), respectively, for stroke prevention. Noted no patients have shifted between different NOACs (e.g. from dabigatran to rivaroxaban, and vice versa) during their whole following period. However, a small number of patients $(n=463$, $4.74 \%$ ) shifted between different dosages of the same NOAC. For the dabigatran group $(n=6,600), 292$ patients had dose alteration (4.52\%): 153 altered from 150 to 110 $\mathrm{mg} ; 139$ altered from 110 to $150 \mathrm{mg}$. For the rivaroxaban group $(n=3,167), 171$ patients had dose alteration $(5.40 \%)$ : 11 and 19 altered from 20 to 15 and $10 \mathrm{mg}$, respectively; 16 and 44 altered from 15 to 20 and $10 \mathrm{mg}$ respectively; 21 and 60 altered from 10 to 20 and $15 \mathrm{mg}$, respectively. For the AA group, no patients have shifted between different AAs or dose adjustment. Since rivaroxaban was only approved after February, 2013 in Taiwan, we selected 4,616 patients from the AA group with a first prescription of AA after February, 2013 for a head-to-head comparison with the rivaroxaban group (Table 2). Before propensity score weighting, dabigatran, rivaroxaban, and warfarin groups were older, had a higher $\mathrm{CHA}_{2} \mathrm{DS}_{2}-\mathrm{VASc}$ score (heart failure, hypertension, age 75 years or older, diabetes mellitus, previous stroke or transient ischemic attack (TIA), vascular disease, age 65 to 74 years, female gender), HAS-BLED score (hypertension, abnormal renal or liver function, stroke, bleeding history, labile INR, age 65 years or older, and antiplatelet drug or alcohol use), and a higher proportion of comorbidities compared to the AA group. The $\mathrm{CHA}_{2} \mathrm{DS}_{2}$-VASc scores were 4.1 $1.6,4.1 \pm 1.6$, $3.3 \pm 1.8$ and $2.4 \pm 1.6$ for the dabigatran, rivaroxaban, warfarin, and AA groups, respectively.

\section{Incidence of bleeding outcome}

The annual incidence of intracranial hemorrhage (ICH) was $0.6 \%, 1.3 \%$, and $0.8 \%$; the risk of gastrointestinal bleeding (GIB) was $1.3 \%, 1.5 \%$, and $1.8 \%$; and the risk of all major bleeding was $1.8 \%$, $2.9 \%$, and $2.6 \%$ for the dabiagtran, warfarin, and AA groups, respectively (Tables 4 and 5). Before weighting, dabigatran was associated with a significantly lower risk of GIB (Hazard ratio (HR): $0.68 ; P=0.0200$ ) and all major bleeding events (HR: $0.67 ; P=0.0040$ ) compared with AA. Warfarin carried a significantly higher risk of ICH compared to AA before adjustment (HR: 1.67; $P=$ 0.0096). After weighting, dabigatran was associated with a significantly lower risk of ICH (HR: $0.36 ; P<0.0001)$, GIB (HR: $0.44 ; P<0.0001)$ and all major bleeding events (HR: $0.41 ; P<0.0001)$ compared with AA. The AA group was further categorized by use of aspirin and $\mathrm{P}_{2} \mathrm{Y}_{12}$ inhibitor (including clopidogrel and ticlopidine). Dabigatran was associated a lower risk of GIB and all major bleeding events compared with either aspirin or $\mathrm{P}_{2} \mathrm{Y}_{12}$ inhibitor (Table 4). Warfarin had a significantly lower risk of GIB (1.53 vs. 2.23\%/year; HR: 0.68; $P=0.0189$ ) than AA after adjustment. The warfarin and AA groups had a similar risk of all major bleeding events $(P=0.4130)$. It is noted that warfarin caused a significantly higher risk of ICH compared with $\mathrm{P}_{2} \mathrm{Y}_{12}$ inhibitor (HR: 3.35; $P<0.0001$ ) (Table 5). The annual incidence of $\mathrm{ICH}$, GIB and all major bleeding was $0.5 \%, 2.0 \%$, and $2.5 \%$ for rivaroxaban, respectively (Table 6). There was no significant difference in the risk of ICH, GIB or all major bleeding for rivaroxaban versus AA before adjustment, whereas rivaroxaban had a significantly lower risk of ICH compared with AA after adjustment (HR: 0.25; $P=0.0037)$. It is noted that rivaroxaban caused a lower risk of all major bleeding compared with $\mathrm{P}_{2} \mathrm{Y}_{12}$ inhibitor (HR: $0.60 ; P=0.0336$ ) (Table 6). Figures 1 and 2 show a clear separation of event curves for ICH, GIB, and allmajor bleeding between the dabigatran and AA groups 
Table 1: Baseline characteristics of non-valvular atrial fibrillation (AF) patients taking dabigatran (D) and antiplatelet agents (AA), before and after propensity score weighting

\begin{tabular}{|c|c|c|c|c|c|c|}
\hline & \multicolumn{6}{|c|}{ Propensity score weighting } \\
\hline & \multicolumn{3}{|c|}{ Before } & \multicolumn{3}{|c|}{ After } \\
\hline & $\begin{array}{c}D \\
(n=6,600)\end{array}$ & $\underset{(n=8,238)}{\text { AA }}$ & ASMD & $\begin{array}{c}D \\
(n=6,600)\end{array}$ & $\underset{(n=8,238)}{\text { AA }}$ & ASMD \\
\hline Age, yrs & $75 \pm 10$ & $68 \pm 14$ & 0.5379 & $75 \pm 10$ & $77 \pm 9$ & 0.1677 \\
\hline$<65$ & $13 \%$ & $40 \%$ & & $13 \%$ & $11 \%$ & \\
\hline $65-74$ & $30 \%$ & $23 \%$ & & $30 \%$ & $25 \%$ & \\
\hline $75-84$ & $42 \%$ & $23 \%$ & & $42 \%$ & $46 \%$ & \\
\hline$>85$ & $15 \%$ & $14 \%$ & & $15 \%$ & $18 \%$ & \\
\hline Male & $58 \%$ & $58 \%$ & 0.0034 & $58 \%$ & $55 \%$ & 0.0658 \\
\hline $\mathrm{CHA}_{2} \mathrm{DS}_{2}-\mathrm{VASc}$ & $4.06 \pm 1.57$ & $2.38 \pm 1.61$ & 1.0520 & $4.06 \pm 1.57$ & $4.43 \pm 1.75$ & 0.2252 \\
\hline HAS-BLED & $3.07 \pm 1.13$ & $2.42 \pm 1.01$ & 0.5988 & $3.07 \pm 1.13$ & $3.71 \pm 1.02$ & 0.5967 \\
\hline Chronic liver disease & $27 \%$ & $11 \%$ & 0.4139 & $27 \%$ & $27 \%$ & 0.0100 \\
\hline Chronic kidney disease & $22 \%$ & $9 \%$ & 0.3514 & $22 \%$ & $24 \%$ & 0.0501 \\
\hline Congestive heart failure & $15 \%$ & $6 \%$ & 0.2828 & $15 \%$ & $20 \%$ & 0.1530 \\
\hline Hypertension & $86 \%$ & $57 \%$ & 0.6614 & $86 \%$ & $88 \%$ & 0.0581 \\
\hline Hyperlipidemia & $51 \%$ & $22 \%$ & 0.6271 & $51 \%$ & $51 \%$ & 0.0092 \\
\hline Diabetes mellitus & $40 \%$ & $20 \%$ & 0.4541 & $40 \%$ & $46 \%$ & 0.1082 \\
\hline Previous stroke & $35 \%$ & $6 \%$ & 0.7585 & $35 \%$ & $42 \%$ & 0.1625 \\
\hline Previous TIA & $5 \%$ & $1 \%$ & 0.2689 & $5 \%$ & $8 \%$ & 0.1208 \\
\hline Myocardial infarction & $2 \%$ & $2 \%$ & 0.0071 & $2 \%$ & $1 \%$ & 0.0749 \\
\hline Gout & $29 \%$ & $11 \%$ & 0.4693 & $29 \%$ & $33 \%$ & 0.0869 \\
\hline Peripheral artery disease & $0 \%$ & $0 \%$ & 0.0302 & $0 \%$ & $0 \%$ & 0.0302 \\
\hline Malignancy & $8 \%$ & $7 \%$ & 0.0406 & $8 \%$ & $9 \%$ & 0.0123 \\
\hline History of bleeding & $2 \%$ & $1 \%$ & 0.0624 & $2 \%$ & $4 \%$ & 0.1338 \\
\hline Use of NSAIDs & $24 \%$ & $27 \%$ & 0.0724 & $24 \%$ & $21 \%$ & 0.0686 \\
\hline Use of PPI & $5 \%$ & $5 \%$ & 0.0125 & $5 \%$ & $4 \%$ & 0.0227 \\
\hline Use of ACEI/ARB & $62 \%$ & $40 \%$ & 0.4413 & $62 \%$ & $60 \%$ & 0.0399 \\
\hline Use of amiodarone & $17 \%$ & $26 \%$ & 0.2253 & $17 \%$ & $14 \%$ & 0.0841 \\
\hline Use of beta-blocker & $50 \%$ & $47 \%$ & 0.0630 & $50 \%$ & $52 \%$ & 0.0290 \\
\hline Use of diltiazem/verapamil & $20 \%$ & $22 \%$ & 0.0299 & $20 \%$ & $19 \%$ & 0.0338 \\
\hline Use of digoxin & $26 \%$ & $18 \%$ & 0.1888 & $26 \%$ & $24 \%$ & 0.0461 \\
\hline Use of statin & $28 \%$ & $12 \%$ & 0.4265 & $28 \%$ & $26 \%$ & 0.0405 \\
\hline PCI & $4 \%$ & $2 \%$ & 0.1178 & $4 \%$ & $2 \%$ & 0.1527 \\
\hline CABG & $1 \%$ & $0 \%$ & 0.0709 & $1 \%$ & $0 \%$ & 0.0796 \\
\hline
\end{tabular}

$\mathrm{ACEI}=$ angiotensin-converting-enzyme inhibitor; $\mathrm{AF}=$ atrial fibrillation; $\mathrm{ARB}=$ angiotensin II receptor antagonists; ASMD $=$ absolute standardized mean difference; $\mathrm{CABG}=$ coronary artery bypass graft; $\mathrm{CHA}_{2} \mathrm{DS}_{2}-\mathrm{VASc}=$ congestive heart failure, hypertension, age 75 years or older, diabetes mellitus, previous stroke/transient ischemic attack, vascular disease, age 65 to 74 years, female; HAS-BLED = hypertension, abnormal renal or liver function, stroke, bleeding history, labile INR, age 65 years or older, and antiplatelet drug or alcohol use. Labile INR could not be determined from claims and was excluded from our scoring; NSAIDs = non-steroid anti-inflammatory drugs; PCI = percutaneous coronary intervention; PPI = proton pump inhibitor; TIA = transient ischemic attack 
Table 2: Baseline characteristics of non-valvular atrial fibrillation (AF) patients taking rivaroxaban (R) and antiplatelet agents (AA), before and after propensity score weighting

\begin{tabular}{|c|c|c|c|c|c|c|}
\hline & \multicolumn{6}{|c|}{ Propensity score weighting } \\
\hline & \multicolumn{3}{|c|}{ Before } & \multicolumn{3}{|c|}{ After } \\
\hline & $\begin{array}{c}\mathrm{R} \\
(\mathrm{n}=\mathbf{3 , 1 6 7 )}\end{array}$ & $\begin{array}{c}\mathrm{AA} \\
(\mathrm{n}=\mathbf{4 , 6 1 6 )} \\
\end{array}$ & ASMD & $\begin{array}{c}\mathrm{R} \\
(\mathrm{n}=\mathbf{3 , 1 6 7 )}\end{array}$ & $\begin{array}{c}\text { AA } \\
(n=4,616) \\
\end{array}$ & ASMD \\
\hline Age, yrs & $76 \pm 9$ & $68 \pm 14$ & 0.6263 & $76 \pm 9$ & $77 \pm 8$ & 0.1064 \\
\hline$<65$ & $11 \%$ & $42 \%$ & & $11 \%$ & $10 \%$ & \\
\hline $65-74$ & $30 \%$ & $22 \%$ & & $30 \%$ & $25 \%$ & \\
\hline $75-84$ & $42 \%$ & $22 \%$ & & $42 \%$ & $48 \%$ & \\
\hline$>85$ & $17 \%$ & $14 \%$ & & $17 \%$ & $17 \%$ & \\
\hline Male & $53 \%$ & $58 \%$ & 0.1069 & $53 \%$ & $51 \%$ & 0.0456 \\
\hline $\mathrm{CHA}_{2} \mathrm{DS}_{2}-\mathrm{VASc}$ & $4.07 \pm 1.61$ & $2.37 \pm 1.63$ & 1.0551 & $4.07 \pm 1.61$ & $4.34 \pm 1.57$ & 0.1663 \\
\hline HAS-BLED & $3.06 \pm 1.14$ & $2.41 \pm 1.02$ & 0.6040 & $3.06 \pm 1.14$ & $3.70 \pm 1.00$ & 0.6017 \\
\hline Chronic liver disease & $27 \%$ & $11 \%$ & 0.4388 & $27 \%$ & $30 \%$ & 0.0633 \\
\hline Chronic kidney disease & $21 \%$ & $9 \%$ & 0.3393 & $21 \%$ & $23 \%$ & 0.0316 \\
\hline Congestive heart failure & $15 \%$ & $6 \%$ & 0.3054 & $15 \%$ & $19 \%$ & 0.0926 \\
\hline Hypertension & $86 \%$ & $57 \%$ & 0.6702 & $86 \%$ & $87 \%$ & 0.0415 \\
\hline Hyperlipidemia & $50 \%$ & $22 \%$ & 0.6162 & $50 \%$ & $53 \%$ & 0.0529 \\
\hline Diabetes mellitus & $39 \%$ & $20 \%$ & 0.4233 & $39 \%$ & $43 \%$ & 0.0880 \\
\hline Previous stroke & $30 \%$ & $6 \%$ & 0.6417 & $30 \%$ & $37 \%$ & 0.1550 \\
\hline Previous TIA & $4 \%$ & $1 \%$ & 0.2298 & $4 \%$ & $6 \%$ & 0.0881 \\
\hline Myocardial infarction & $3 \%$ & $2 \%$ & 0.0553 & $3 \%$ & $1 \%$ & 0.0997 \\
\hline Gout & $28 \%$ & $10 \%$ & 0.4720 & $28 \%$ & $32 \%$ & 0.1041 \\
\hline Peripheral artery disease & $0 \%$ & $0 \%$ & 0.0000 & $0 \%$ & $0 \%$ & 0.0000 \\
\hline Malignancy & $9 \%$ & $7 \%$ & 0.0560 & $9 \%$ & $8 \%$ & 0.0137 \\
\hline History of bleeding & $2 \%$ & $1 \%$ & 0.0868 & $2 \%$ & $5 \%$ & 0.1556 \\
\hline Use of NSAIDs & $22 \%$ & $27 \%$ & 0.1239 & $22 \%$ & $20 \%$ & 0.0628 \\
\hline Use of PPI & $7 \%$ & $5 \%$ & 0.0540 & $7 \%$ & $7 \%$ & 0.0025 \\
\hline Use of ACEI/ARB & $60 \%$ & $40 \%$ & 0.4015 & $60 \%$ & $59 \%$ & 0.0331 \\
\hline Use of amiodarone & $16 \%$ & $26 \%$ & 0.2500 & $16 \%$ & $13 \%$ & 0.0663 \\
\hline Use of beta-blocker & $52 \%$ & $48 \%$ & 0.0882 & $52 \%$ & $53 \%$ & 0.0049 \\
\hline Use of diltiazem/verapamil & $21 \%$ & $21 \%$ & 0.0104 & $21 \%$ & $22 \%$ & 0.0197 \\
\hline Use of digoxin & $26 \%$ & $18 \%$ & 0.2009 & $26 \%$ & $26 \%$ & 0.0100 \\
\hline Use of statin & $28 \%$ & $12 \%$ & 0.4031 & $28 \%$ & $25 \%$ & 0.0635 \\
\hline PCI & $5 \%$ & $2 \%$ & 0.1785 & $5 \%$ & $2 \%$ & 0.1864 \\
\hline CABG & $1 \%$ & $0 \%$ & 0.0827 & $1 \%$ & $0 \%$ & 0.0823 \\
\hline
\end{tabular}

The abbreviations as in Table 1. 
Table 3: Baseline characteristics of non-valvular atrial fibrillation (AF) patients taking warfarin (W) and antiplatelet agents (AA), before and after propensity score weighting

\begin{tabular}{|c|c|c|c|c|c|c|}
\hline & \multicolumn{6}{|c|}{ Propensity score weighting } \\
\hline & \multicolumn{3}{|c|}{ Before } & \multicolumn{3}{|c|}{ After } \\
\hline & $\begin{array}{c}W \\
(n=5,338)\end{array}$ & $\begin{array}{c}\text { AA } \\
(n=8,238)\end{array}$ & ASMD & $\begin{array}{c}W \\
(n=5,338)\end{array}$ & $\begin{array}{c}\text { AA } \\
(n=8,238)\end{array}$ & ASMD \\
\hline Age, yrs & $71 \pm 12$ & $68 \pm 14$ & 0.1809 & $71 \pm 12$ & $70 \pm 10$ & 0.0292 \\
\hline$<65$ & $31 \%$ & $40 \%$ & & $31 \%$ & $32 \%$ & \\
\hline $65-74$ & $27 \%$ & $23 \%$ & & $27 \%$ & $26 \%$ & \\
\hline $75-84$ & $29 \%$ & $23 \%$ & & $29 \%$ & $29 \%$ & \\
\hline$>85$ & $13 \%$ & $14 \%$ & & $13 \%$ & $13 \%$ & \\
\hline Male & $55 \%$ & $58 \%$ & 0.0720 & $55 \%$ & $53 \%$ & 0.0231 \\
\hline $\mathrm{CHA}_{2} \mathrm{DS}_{2}-\mathrm{VASc}$ & $3.25 \pm 1.80$ & $2.38 \pm 1.61$ & 0.5088 & $3.25 \pm 1.80$ & $3.26 \pm 1.49$ & 0.0051 \\
\hline HAS-BLED & $2.61 \pm 1.33$ & $2.42 \pm 1.01$ & 0.1538 & $2.61 \pm 1.33$ & $3.06 \pm 0.94$ & 0.3927 \\
\hline Chronic liver disease & $22 \%$ & $11 \%$ & 0.3004 & $22 \%$ & $22 \%$ & 0.0020 \\
\hline Chronic kidney disease & $19 \%$ & $9 \%$ & 0.2913 & $19 \%$ & $20 \%$ & 0.0165 \\
\hline Congestive heart failure & $14 \%$ & $6 \%$ & 0.2256 & $14 \%$ & $14 \%$ & 0.0069 \\
\hline Hypertension & $74 \%$ & $57 \%$ & 0.3546 & $74 \%$ & $74 \%$ & 0.0018 \\
\hline Hyperlipidemia & $40 \%$ & $22 \%$ & 0.3972 & $40 \%$ & $40 \%$ & 0.0116 \\
\hline Diabetes mellitus & $33 \%$ & $20 \%$ & 0.3007 & $33 \%$ & $33 \%$ & 0.0051 \\
\hline Previous stroke & $20 \%$ & $6 \%$ & 0.4364 & $20 \%$ & $22 \%$ & 0.0313 \\
\hline Previous TIA & $2 \%$ & $1 \%$ & 0.1370 & $2 \%$ & $3 \%$ & 0.0102 \\
\hline Myocardial infarction & $1 \%$ & $2 \%$ & 0.0530 & $1 \%$ & $1 \%$ & 0.0417 \\
\hline Gout & $22 \%$ & $11 \%$ & 0.2986 & $22 \%$ & $22 \%$ & 0.0098 \\
\hline Peripheral artery disease & $0 \%$ & $0 \%$ & 0.0000 & $0 \%$ & $0 \%$ & 0.0000 \\
\hline Malignancy & $8 \%$ & $7 \%$ & 0.0207 & $8 \%$ & $8 \%$ & 0.0082 \\
\hline History of bleeding & $2 \%$ & $1 \%$ & 0.0730 & $2 \%$ & $3 \%$ & 0.0168 \\
\hline Use of NSAIDs & $27 \%$ & $27 \%$ & 0.0159 & $27 \%$ & $26 \%$ & 0.0075 \\
\hline Use of PPI & $7 \%$ & $5 \%$ & 0.0828 & $7 \%$ & $8 \%$ & 0.0168 \\
\hline Use of ACEI/ARB & $56 \%$ & $40 \%$ & 0.3248 & $56 \%$ & $57 \%$ & 0.0139 \\
\hline Use of amiodarone & $29 \%$ & $26 \%$ & 0.0721 & $29 \%$ & $28 \%$ & 0.0318 \\
\hline Use of beta-blocker & $55 \%$ & $47 \%$ & 0.1670 & $55 \%$ & $56 \%$ & 0.0037 \\
\hline Use of diltiazem/verapamil & $23 \%$ & $22 \%$ & 0.0305 & $23 \%$ & $22 \%$ & 0.0232 \\
\hline Use of digoxin & $27 \%$ & $18 \%$ & 0.2201 & $27 \%$ & $27 \%$ & 0.0105 \\
\hline Use of statin & $20 \%$ & $12 \%$ & 0.2359 & $20 \%$ & $19 \%$ & 0.0177 \\
\hline PCI & $2 \%$ & $2 \%$ & 0.0010 & $2 \%$ & $1 \%$ & 0.0674 \\
\hline CABG & $0 \%$ & $0 \%$ & 0.0620 & $0 \%$ & $0 \%$ & 0.0360 \\
\hline
\end{tabular}

The abbreviations as in Table 1. 
Table 4: Incidence (per 100 person-years) of bleeding events in patients with non-valvular atrial fibrillation (AF) receiving dabigatran (D) and antiplatelet agents (AA)

\begin{tabular}{|c|c|c|c|c|c|c|c|c|c|c|c|c|}
\hline & \multicolumn{4}{|c|}{ Intracranial hemorrhage } & \multicolumn{4}{|c|}{ Gastrointestinal bleeding } & \multicolumn{4}{|c|}{ All major bleeding } \\
\hline & $\begin{array}{l}\text { Crude } \\
\text { events }\end{array}$ & $\begin{array}{c}\text { Crude } \\
\text { incidence }\end{array}$ & $\begin{array}{c}\text { Adjusted } \\
\text { events }\end{array}$ & $\begin{array}{l}\text { Adjusted } \\
\text { incidence }\end{array}$ & $\begin{array}{l}\text { Crude } \\
\text { events }\end{array}$ & $\begin{array}{c}\text { Crude } \\
\text { incidence }\end{array}$ & $\begin{array}{c}\text { Adjusted } \\
\text { events }\end{array}$ & $\begin{array}{l}\text { Adjusted } \\
\text { incidence }\end{array}$ & $\begin{array}{l}\text { Crude } \\
\text { events }\end{array}$ & $\begin{array}{c}\text { Crude } \\
\text { incidence }\end{array}$ & $\begin{array}{c}\text { Adjusted }^{*} \\
\text { events }\end{array}$ & $\begin{array}{l}\text { Adjusted* } \\
\text { incidence }\end{array}$ \\
\hline \multicolumn{13}{|c|}{ (1) D versus AA } \\
\hline D & 24 & 0.55 & 24 & 0.55 & 55 & 1.25 & 55 & 1.25 & 79 & 1.80 & 79 & 1.80 \\
\hline$(n=6,600)$ & & $\begin{array}{c}(0.33- \\
0.77)\end{array}$ & & $\begin{array}{c}(0.33- \\
0.77)\end{array}$ & & $\begin{array}{c}(0.92- \\
1.58)\end{array}$ & & $\begin{array}{l}(0.92- \\
1.58)\end{array}$ & & $\begin{array}{l}(1.40- \\
2.20)\end{array}$ & & $\begin{array}{l}(1.40- \\
2.20)\end{array}$ \\
\hline $\mathbf{A A}$ & 50 & 0.76 & 82.08 & 1.47 & 115 & 1.76 & 159.32 & 2.86 & 167 & 2.55 & 242 & 4.34 \\
\hline$(n=8,238)$ & & $\begin{array}{l}(0.22- \\
0.98)\end{array}$ & & $\begin{array}{c}(1.15- \\
1.79)\end{array}$ & & $\begin{array}{l}(1.44- \\
2.08)\end{array}$ & & $\begin{array}{c}(2.41- \\
3.30)\end{array}$ & & $\begin{array}{l}(2.16- \\
2.94)\end{array}$ & & $\begin{array}{l}(3.80- \\
4.90)\end{array}$ \\
\hline $\begin{array}{l}\text { D vs. AA } \\
\text { HR }\end{array}$ & & 0.68 & & 0.36 & & 0.68 & & 0.44 & & 0.67 & & 0.41 \\
\hline$(95 \%$ CI) & & $\begin{array}{c}(0.42- \\
1.11)\end{array}$ & & $\begin{array}{c}(0.23- \\
0.57)\end{array}$ & & $\begin{array}{c}(0.50- \\
0.94)\end{array}$ & & $\begin{array}{l}(0.32- \\
0.59)\end{array}$ & & $\begin{array}{l}(0.52- \\
0.88)\end{array}$ & & $\begin{array}{l}(0.32- \\
0.53)\end{array}$ \\
\hline Pvalue & & 0.1233 & & $<0.0001$ & & 0.0200 & & $<0.0001$ & & 0.0040 & & $<0.0001$ \\
\hline (2) Aspirin & 46 & 0.77 & 97.19 & 1.72 & 89 & 1.49 & 176.44 & 3.13 & 137 & 2.29 & 274.60 & 4.87 \\
\hline$(n=7,516)$ & & $\begin{array}{c}(0.55- \\
0.99)\end{array}$ & & $\begin{array}{c}(1.38- \\
2.07)\end{array}$ & & $\begin{array}{c}(1.18- \\
1.78)\end{array}$ & & $\begin{array}{l}(2.67- \\
3.59)\end{array}$ & & $\begin{array}{l}(1.91- \\
2.68)\end{array}$ & & $\begin{array}{c}(4.29- \\
5.44)\end{array}$ \\
\hline $\begin{array}{l}\text { D vs. } \\
\text { Aspirin }\end{array}$ & & 0.68 & & 0.31 & & 0.80 & & 0.40 & & 0.75 & & 0.37 \\
\hline HR & & & & & & & & & & & & \\
\hline (95\% CI) & & $\begin{array}{c}(0.41- \\
1.11)\end{array}$ & & $\begin{array}{c}(0.20- \\
0.49)\end{array}$ & & $\begin{array}{l}(0.57- \\
1.12)\end{array}$ & & $\begin{array}{c}(0.30- \\
0.54)\end{array}$ & & $\begin{array}{c}(0.57- \\
0.99)\end{array}$ & & $\begin{array}{c}(0.29- \\
0.47)\end{array}$ \\
\hline Pvalue & & 0.1216 & & $<0.0001$ & & 0.2009 & & $<0.0001$ & & 0.0413 & & $<0.0001$ \\
\hline $\begin{array}{l}\text { (3) } P_{2} Y_{12} \\
\text { inhibitor }\end{array}$ & 9 & 1.08 & 26.20 & 0.49 & 31 & 3.73 & 130.37 & 2.45 & 40 & 4.82 & 156.57 & 2.95 \\
\hline$(\mathrm{n}=\mathbf{1 , 0 5 7})$ & & $\begin{array}{l}(0.50- \\
2.06)\end{array}$ & & $\begin{array}{c}(0.30- \\
0.68)\end{array}$ & & $\begin{array}{c}(2.42- \\
5.05)\end{array}$ & & $\begin{array}{l}(2.03- \\
2.87)\end{array}$ & & $\begin{array}{c}(3.33- \\
6.31)\end{array}$ & & $\begin{array}{l}(2.48- \\
3.41)\end{array}$ \\
\hline $\begin{array}{l}\text { D vs. } P_{2} Y_{12} \\
\text { inhibitor }\end{array}$ & & 0.48 & & 1.07 & & 0.32 & & 0.50 & & 0.36 & & 0.59 \\
\hline HR & & & & & & & & & & & & \\
\hline$(95 \%$ CI $)$ & & $\begin{array}{c}(0.22- \\
1.03)\end{array}$ & & $\begin{array}{c}(0.61- \\
1.86)\end{array}$ & & $\begin{array}{c}(0.21- \\
0.50)\end{array}$ & & $\begin{array}{c}(0.36- \\
0.68)\end{array}$ & & $\begin{array}{c}(0.24- \\
0.52)\end{array}$ & & $\begin{array}{c}(0.45- \\
0.78)\end{array}$ \\
\hline Pvalue & & 0.0601 & & 0.8155 & & $<0.0001$ & & $<0.0001$ & & $<0.0001$ & & 0.0002 \\
\hline
\end{tabular}

$\mathrm{CI}=$ confidential interval; $\mathrm{HR}=$ hazard ratio

${ }^{*}$ event numbers and incidence of antiplatelet agent versus dabigatran after propensity score weighting

both before as well as after propensity score weighting adjustment.

\section{Predictors of major bleeding}

Multivariable analysis showed that age $\geq$ 75 years was statistically significant independent predictor of major bleeding in the dabigatran, warfarin as well as AA groups $(P<0.01$ in all groups). Presence of heart failure (HR, 1.85; $P=0.0195)$ and chronic kidney disease (HR, 2.28; $P=0.0005$ ) were the other independent predictors of major bleeding events in the dabigatran group. Additionally, the presence of stroke/TIA and use of non-steroid anti-inflammatory 
Table 5: Incidence (per 100 person-years) of bleeding events in patients with non-valvular atrial fibrillation (AF) receiving warfarin (W) and antiplatelet agents (AA)

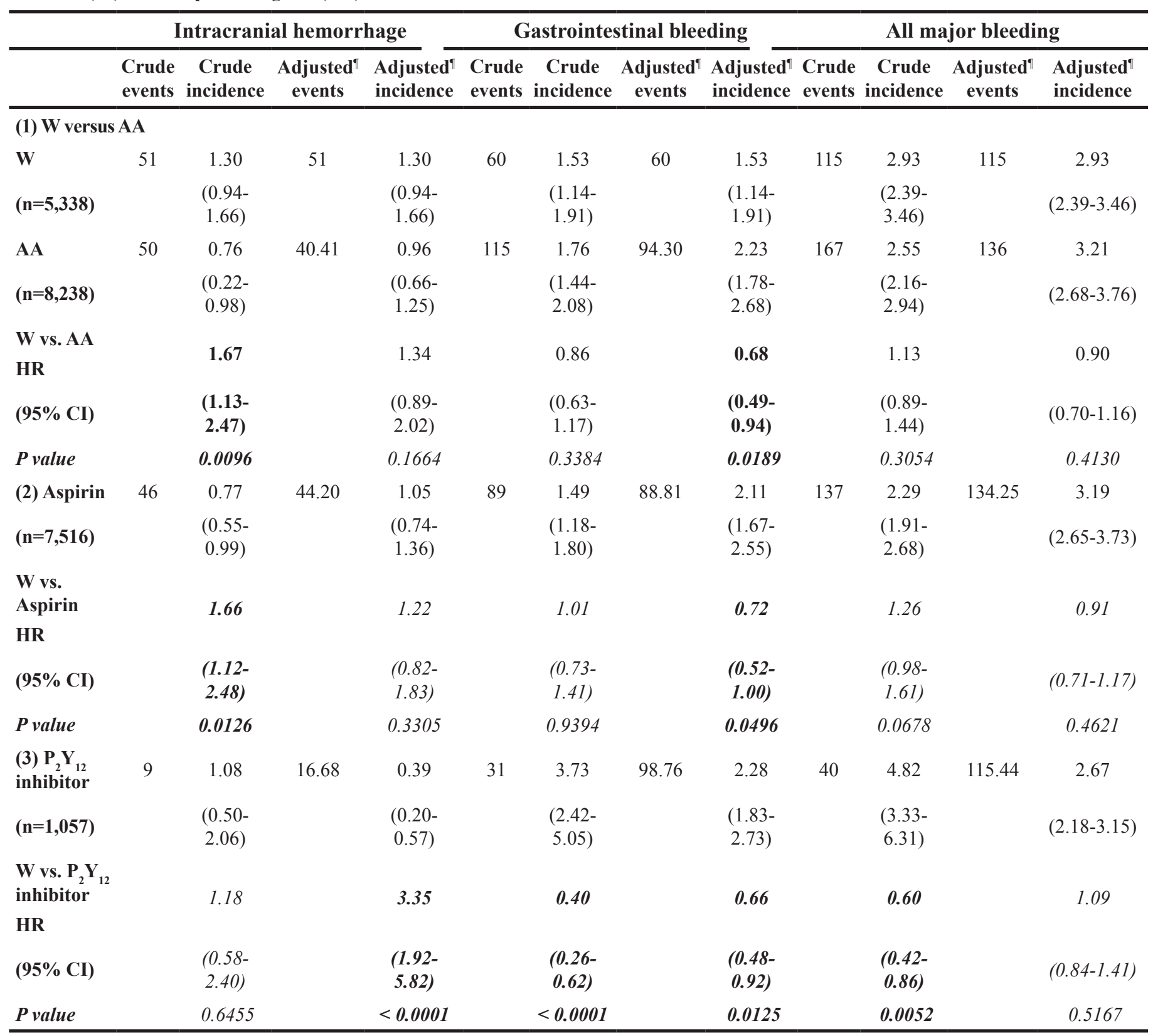

$\mathrm{CI}=$ confidential interval; $\mathrm{HR}=$ hazard ratio

" event numbers and incidence of antiplatelet agent versus warfarin after propensity score weighting

drugs were significant independent predictors of major bleeding in the AA and warfarin groups $(P<0.05$ in both groups). Female and use of non-steroid antiinflammatory drugs were significant independent predictors of major bleeding in the rivaroxaban group (Table 7).

\section{Bleeding risk in selected subgroups}

Subgroup analysis was performed to determine whether patients in the dabigatran and warfarin groups had a lower risk of all major bleeding compared to patients taking either aspirin or $\mathrm{P}_{2} \mathrm{Y}_{12}$ inhibitor 
Table 6: Incidence (per 100 person-years) of bleeding events in patients with non-valvular atrial fibrillation (AF) receiving rivaroxaban $(\mathrm{R})$ and antiplatelet agents (AA)

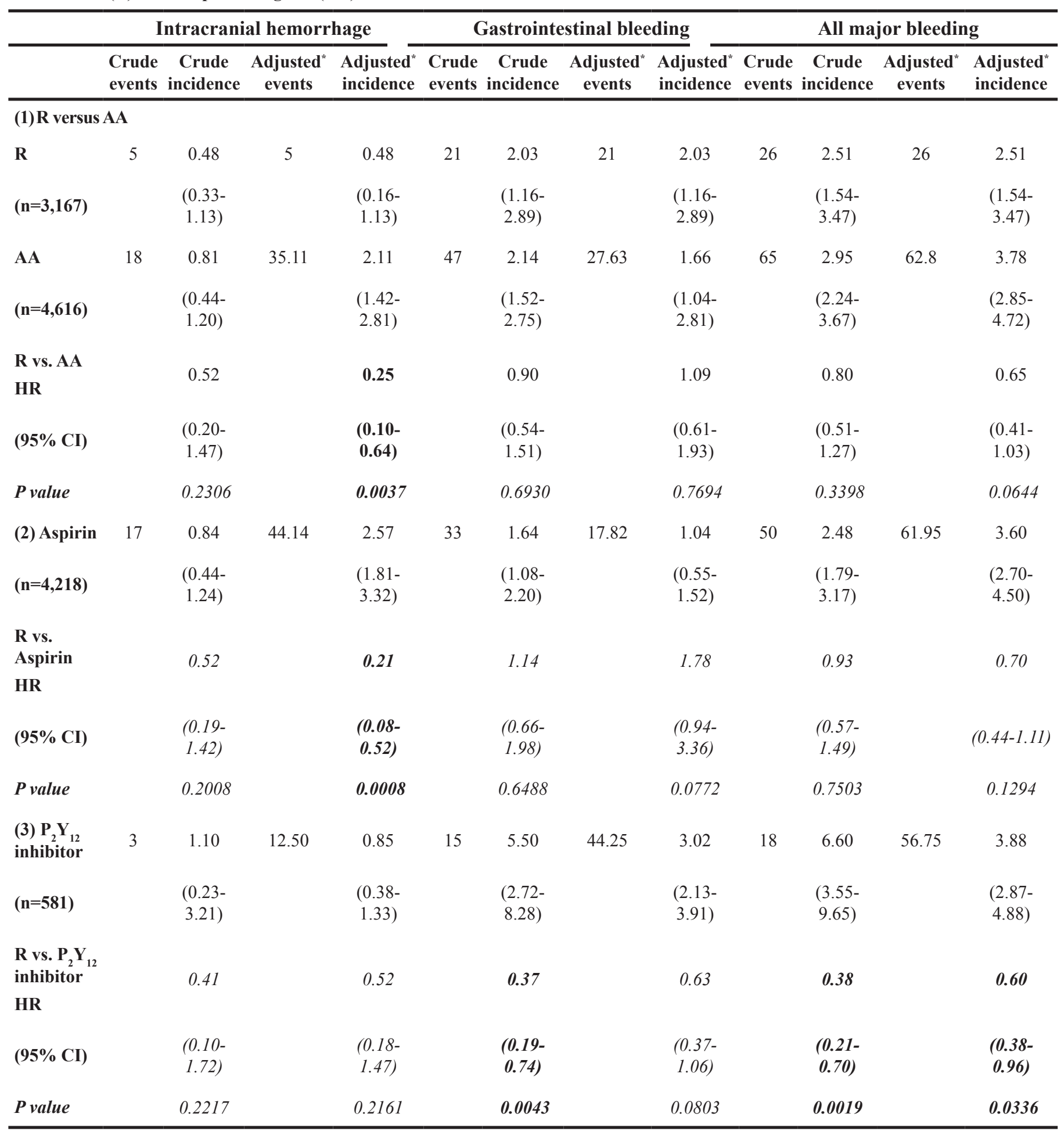

$\mathrm{CI}=$ confidential interval; $\mathrm{HR}=$ hazard ratio

* event numbers and incidence of antiplatelet agent versus rivaroxaban after propensity score weighting 
Table 7: Multivariable analysis of predictors of all major bleeding in 4 treatment groups

\begin{tabular}{|c|c|c|c|c|}
\hline & $\begin{array}{c}\text { Dabigatran } \\
(n=6,600) \\
\text { Hazard ratio } \\
(95 \% \text { CI }) ; P \text {-value }\end{array}$ & $\begin{array}{c}\text { Rivaroxaban } \\
(\mathrm{n}=3,167) \\
\text { Hazard ratio } \\
(95 \% \text { CI }) ; P \text {-value }\end{array}$ & $\begin{array}{c}\text { Warfarin }(\mathrm{n}=5,338) \\
\text { Hazard ratio } \\
(95 \% \mathrm{CI}) ; P \text {-value }\end{array}$ & $\begin{array}{l}\text { Antiplatelet agent } \\
(n=8,238) \\
\text { Hazard ratio } \\
(95 \% \text { CI }) ; P \text {-value }\end{array}$ \\
\hline Age $>=75$ & $\begin{array}{c}2.13(1.27-3.59) \\
0.0043\end{array}$ & $\begin{array}{c}2.15(0.85-5.45) \\
0.1056\end{array}$ & $\begin{array}{c}2.89(1.90-4.39) \\
<0.0001\end{array}$ & $\begin{array}{c}3.09(2.19-4.34) \\
<0.0001\end{array}$ \\
\hline Male & $\begin{array}{c}1.14(0.72-1.80) \\
0.5739\end{array}$ & $\begin{array}{c}0.41(0.18-0.97) \\
0.0421\end{array}$ & $1.00(0.69-1.45) ; 0.9877$ & $1.61(0.85-1.59) ; 0.3493$ \\
\hline Stroke/TIA & $\begin{array}{c}1.43(0.92-2.24) \\
0.1148\end{array}$ & $\begin{array}{c}1.37(0.61-3.05) \\
0.8769\end{array}$ & $1.51(1.01-2.24) ; 0.0443$ & $\begin{array}{c}\text { 1.82(1.17-2.82) } \\
0.0079\end{array}$ \\
\hline Hypertension & $\begin{array}{c}1.57(0.67-3.66) \\
0.3009\end{array}$ & $\begin{array}{c}0.91(0.26-3.16) \\
0.8769\end{array}$ & $1.22(0.73-2.05) ; 0.4445$ & $\begin{array}{c}\text { 1.47(1.04-2.10); } \\
0.0315\end{array}$ \\
\hline $\begin{array}{l}\text { Congestive } \\
\text { heart failure }\end{array}$ & $\begin{array}{c}\text { 1.85(1.10-3.09); } \\
0.0195\end{array}$ & $\begin{array}{c}1.22(0.46-3.28) \\
0.6881\end{array}$ & $1.47(0.92-2.35) ; 0.1054$ & $1.04(0.59-1.80) ; 0.9031$ \\
\hline $\begin{array}{l}\text { Diabetes } \\
\text { mellitus }\end{array}$ & $\begin{array}{c}0.68(0.43-1.10) \\
0.1150\end{array}$ & $\begin{array}{c}0.89(0.39-2.02) \\
0.7789\end{array}$ & $0.90(0.60-1.36) ; 0.6168$ & $1.05(0.73-1.51) ; 0.7877$ \\
\hline Malignancy & $\begin{array}{c}1.14(0.55-2.37) \\
0.7311\end{array}$ & $\begin{array}{c}0.46(0.06-3.38) \\
0.4418\end{array}$ & $1.34(0.76-2.43) ; 0.3025$ & $1.42(0.88-2.27) ; 0.1478$ \\
\hline $\begin{array}{l}\text { Chronic } \\
\text { kidney } \\
\text { disease }\end{array}$ & $\begin{array}{c}2.28(1.43-3.64) \\
0.0005\end{array}$ & $\begin{array}{c}1.06(0.41-2.73) \\
0.9016\end{array}$ & $1.39(0.90-2.13) ; 0.1346$ & $\begin{array}{c}\text { 1.77(1.18-2.66); } \\
0.0062\end{array}$ \\
\hline $\begin{array}{l}\text { Chronic liver } \\
\text { disease }\end{array}$ & $\begin{array}{c}1.07(0.66-1.75) \\
0.7809\end{array}$ & $\begin{array}{c}0.98(0.40-2.42) \\
0.9682\end{array}$ & $0.75(0.46-1.22) ; 0.2452$ & $0.80(0.48-1.34) ; 0.3966$ \\
\hline $\begin{array}{l}\text { Use of } \\
\text { NSAIDs }\end{array}$ & $\begin{array}{c}1.51(0.94-2.42) \\
0.0879\end{array}$ & $\begin{array}{c}2.31(1.04-5.16) \\
0.0410\end{array}$ & $1.78(1.22-2.60) ; 0.0026$ & $\begin{array}{c}\text { 1.75(1.28-2.39) } \\
0.0005\end{array}$ \\
\hline
\end{tabular}

The abbreviations as in Table 1

(including clopidogrel and ticlopidine). A total of 5,822 $(88 \%)$ and $164(5 \%)$ patients were prescribed low dose dabigatran (110 $\mathrm{mg}$ twice daily) and rivaroxaban (10 $\mathrm{mg}$ once daily), respectively. Among these patients, there were 3,846 (58\%) and 2,046 (65\%) patients with a previous history of warfarin exposure, respectively. Our analysis showed patients on dabigatran had a lower risk of major bleeding compared to patients with either aspirin (Figure 3) or $\mathrm{P}_{2} \mathrm{Y}_{12}$ inhibitor (Figure 4) in most subgroups. In contrast to patients in the dabigatran group, patients in the rivaroxaban and warfarin groups had a similar risk of major bleeding compared to either aspirin or $\mathrm{P}_{2} \mathrm{Y}_{12}$ inhibitor group in most subgroups (Figures 5 to 8 ).

\section{DISCUSSION}

This large population-based study investigated the safety profiles of dabigatran, rivaroxaban, and warfarin versus AA with a specific focus on Asians with NVAF taking antithrombotic agents. Our data showed that nearly $90 \%$ of patients taking low doses of dabigatran (110 mg twice daily) had a significantly lower risk of ICH, GIB and all major bleeding compared to patients taking AA after adjustment. Importantly, patients in the warfarin and AA groups had a similar risk of $\mathrm{ICH}$ and all major bleeding after adjustment. Approximately $87 \%$ of patients taking low doses of rivaroxaban (10 or $15 \mathrm{mg}$ once daily) had a 
significantly lower risk of $\mathrm{ICH}$, but a similar risk of GIB and all major bleeding compared to patients in the AA group after adjustment. Subgroup analysis confirmed that dabigatran was associated with a lower risk of major bleeding compared with AA in most subgroups, whereas warfarin and rivaroxaban were associated with a similar risk of major bleeding compared with AA in most subgroups.

Aspirin has been shown to be ineffective for stroke prevention among whites with AF. [1, 13] Recent data from a Japanese trial and a Hong Kong cohort also indicated that aspirin was not effective for stroke prevention among Asians with AF. $[14,15]$ It is estimated that as high as $25 \%$ of AF patients take aspirin for stroke prevention in Asia, possibly due to an impression that aspirin is safer than warfarin. [16] There are several explanations for the unique phenomenon of warfarin underutilization in Asia: It has been reported a 2 to 4-fold higher risk of ICH in Asians treated with warfarin compared with whites over the entire therapeutic range. $[4,5]$ It seemed that it is more difficult to maintain the therapeutic range of international normalized ratio (INR) of 2 to 3 among Asians on warfarin compared with nonAsians, possibly due to ethnicity and drug interaction. $[6,17]$ For the above reasons, physicians were relatively reluctant to prescribe warfarin in Asia.

The BAFTA and recent Hong Kong cohort studies indicated there was no difference in the risk of
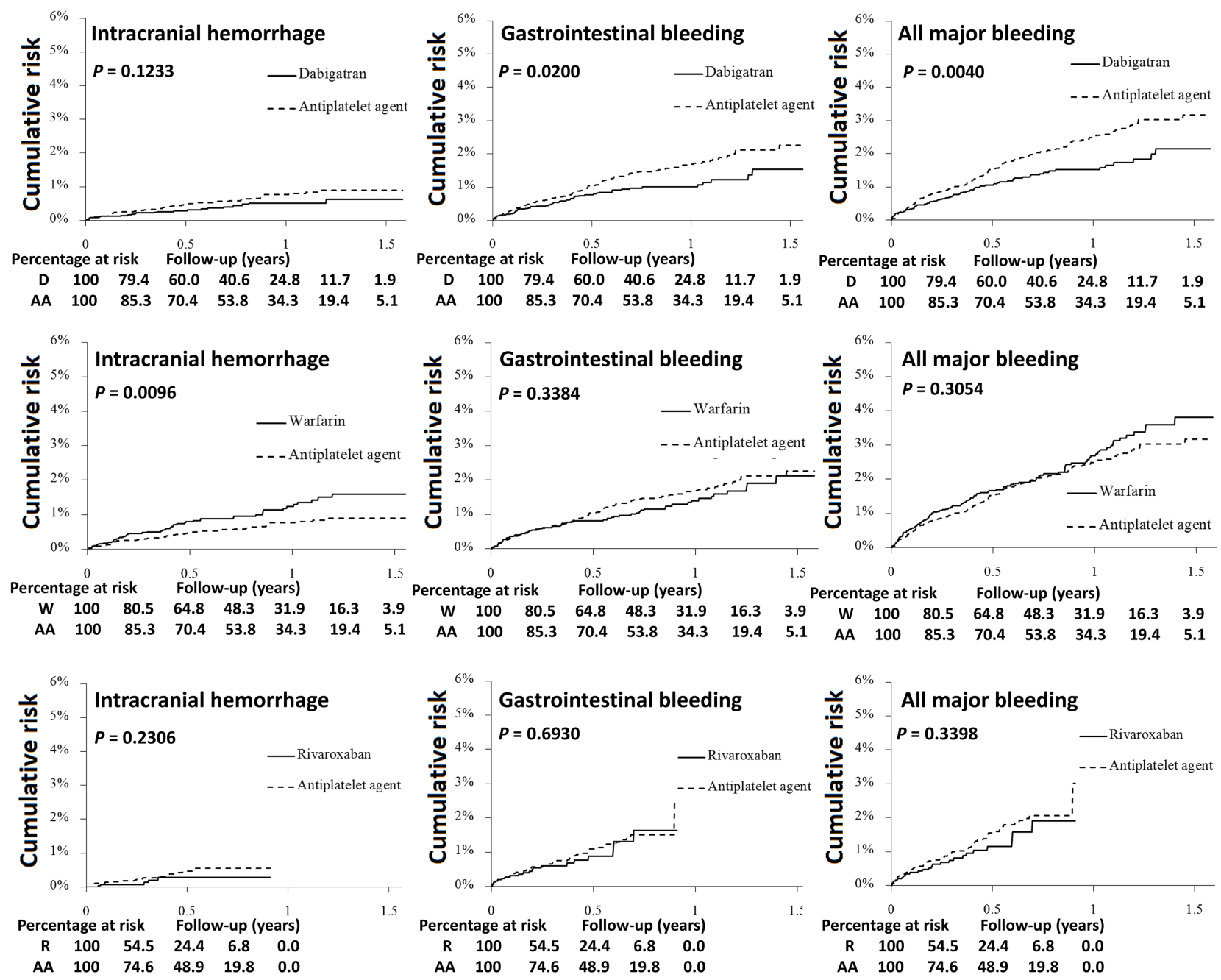

Figure 1: Cumulative risk of intracranial hemorrhage, gastrointestinal bleeding, and all major bleeding for nonvalvular AF (NVAF) patients treated with dabigatran, warfarin or rivaroxaban versus AA before propensity score weighting. Patients on dabigatran (solid line) have a lower risk of gastrointestinal bleeding and all major bleeding than those on antiplatelet agents (dotted line). Patients on warfarin (solid line) have a higher risk of intracranial hemorrhage than those on antiplatelet agents (dotted line). Patients on rivaroxaban (solid line) have a similar risk of intracranial hemorrhage, gastrointestinal bleeding and all major bleeding than those on antiplatelet agents (dotted line). 
cerebrovascular hemorrhage between warfarin and aspirin. $[13,15,23]$ The 2016 ESC guidelines for AF reported that the risk of major bleeding or $\mathrm{ICH}$ associated with aspirin is not significantly different from that of oral anticoagulants, which was mainly based on results from the BAFTA study. [19] In the contrast, a recent meta-analysis which pooled several randomized trials investigating the major bleeding risk associated with aspirin versus vitamin $\mathrm{K}$ antagonists (VKA), including the recently published WARCEF trial, indicated that the risk of major bleeding was substantially higher for VKA targeting the current usual INR range compared to aspirin. [20] Our study showed that the annual risk of $\mathrm{ICH}$ in our Asian cohort taking warfarin was $1.3 \%$, which was compatible to the reported risk of 1.1 to $2.5 \%$ in the Asian subgroup analyses from the four pivotal NOACs trials. [21, 22] Although the risk of ICH in the warfarin group was significantly higher compared to the AA group $(0.76 \%)$ before adjustment, warfarin did not cause a higher ICH risk than AA after co-morbidity adjustment. Additionally, our study indicated that AA use was indeed associated with a higher risk of GIB compared with warfarin. Considering the similar incidence of overall major bleeding events between the warfarin and AA groups either before or after adjustment, our study concluded that AA was not a "safer" alternative to warfarin in reducing major bleeding events in Asians.
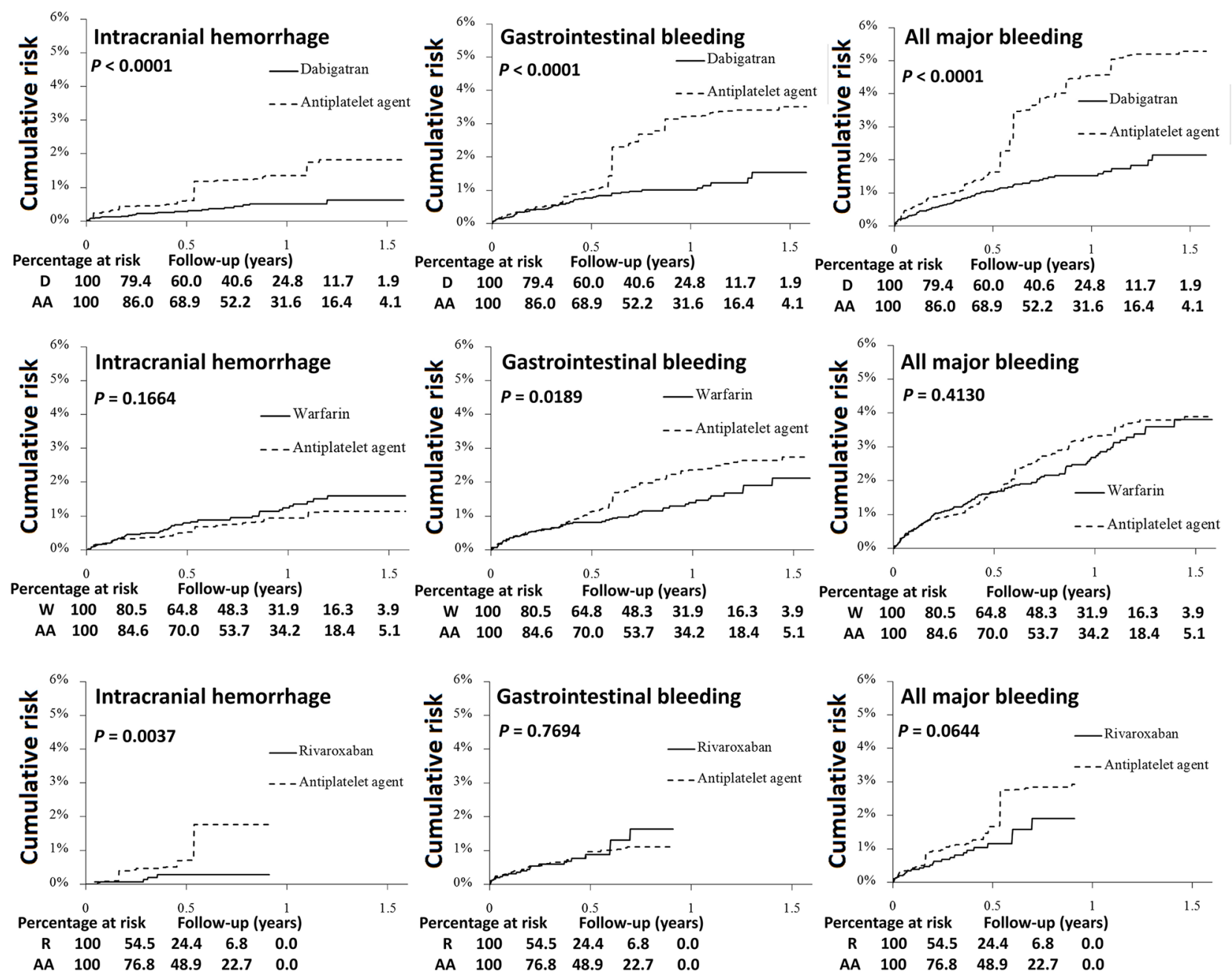

Figure 2: Cumulative risk of intracranial hemorrhage, gastrointestinal bleeding, and all major bleeding for NVAF patients treated with dabigatran, warfarin, or rixaroxaban versus AA after propensity score weighting. Patients on dabigatran (solid line) had a lower risk of intracranial hemorrhage, gastrointestinal bleeding, and all major bleeding compared to those on antiplatelet agents (dotted line). Patients on warfarin (solid line) had a lower risk of gastrointestinal bleeding, and a similar risk of intracranial hemorrhage and all major bleeding compared to those on antiplatelet agents (dotted line). Patients on rivaroxabn (solid line) had a lower risk of intracranial hemorrhage, and a similar risk of gastrointestinal bleeding and all major bleeding compared to those on antiplatelet agents (dotted line). 
There are few studies which directly compared the bleeding risk between NOACs and aspirin. Until now, only the AVERROES trial reported that apixaban was superior to aspirin for stroke prevention in AF patients who were deemed unsuitable for warfarin treatment, and apixaban reduced the risk of stroke/systemic embolism from $3.7 \%$ /year to $1.6 \%$ /year compared with aspirin. [7] There was no significant difference in major bleeding risk between apixaban and aspirin (1.4\%/year versus $1.2 \%$ /year). Ho et al. investigated the $\mathrm{ICH}$ risk in a real-world cohort of Chinese AF patients receiving warfarin, dabigatran, aspirin, or no therapy. $[17,18]$ The incidence of ICH was lowest in patients on dabigatran $(0.32 \%$ /year $)$ as compared with those on aspirin $(0.80 \%$ / year). However, the patient numbers in this study were very limited, with only 393 and 3,600 patients taking dabigatran and aspirin, respectively. In contrast, our study is the largest ever examination of the safety of anti-

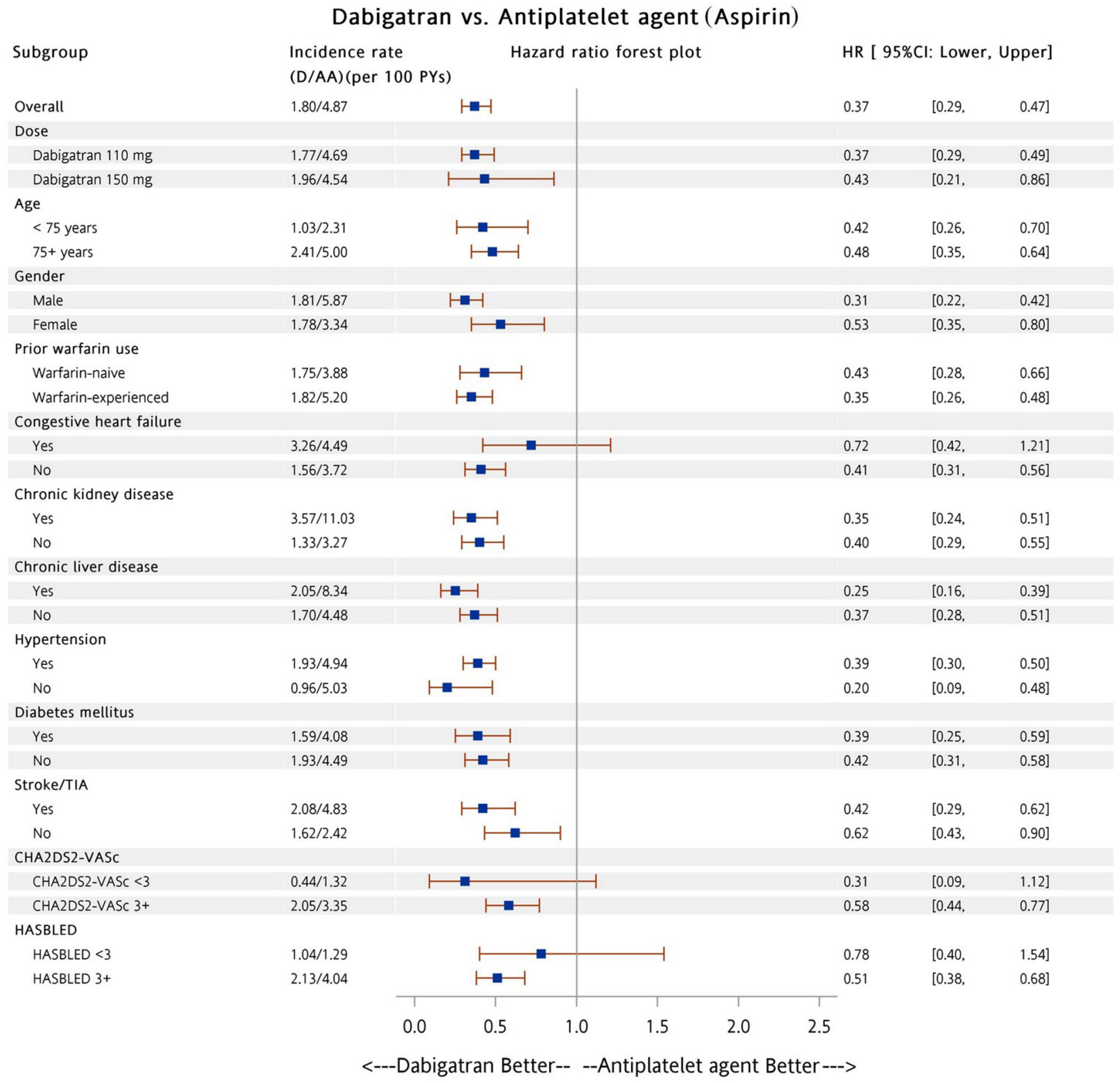

Abbreviation: $\mathrm{D}$, dabigatran; $\mathrm{AA}$, antiplatelet agent, $\mathrm{HR}$, hazard ratio, $\mathrm{Cl}$, confidence interval.

Figure 3: Forest plot of hazard ratio of all major bleeding for NVAF patients taking dabigatran versus aspirin after propensity score weighting. Dabigatran was associated with a lower risk of all major bleeding events compared with aspirin in most subgroups. The abbreviations as in Figure 1. 
Table 8: International Classification of Disease $\left(9^{\text {th }}\right.$ edition) Clinical Modification (ICD 9-CM) codes used to define the co-morbidities and clinical outcome in the study cohort

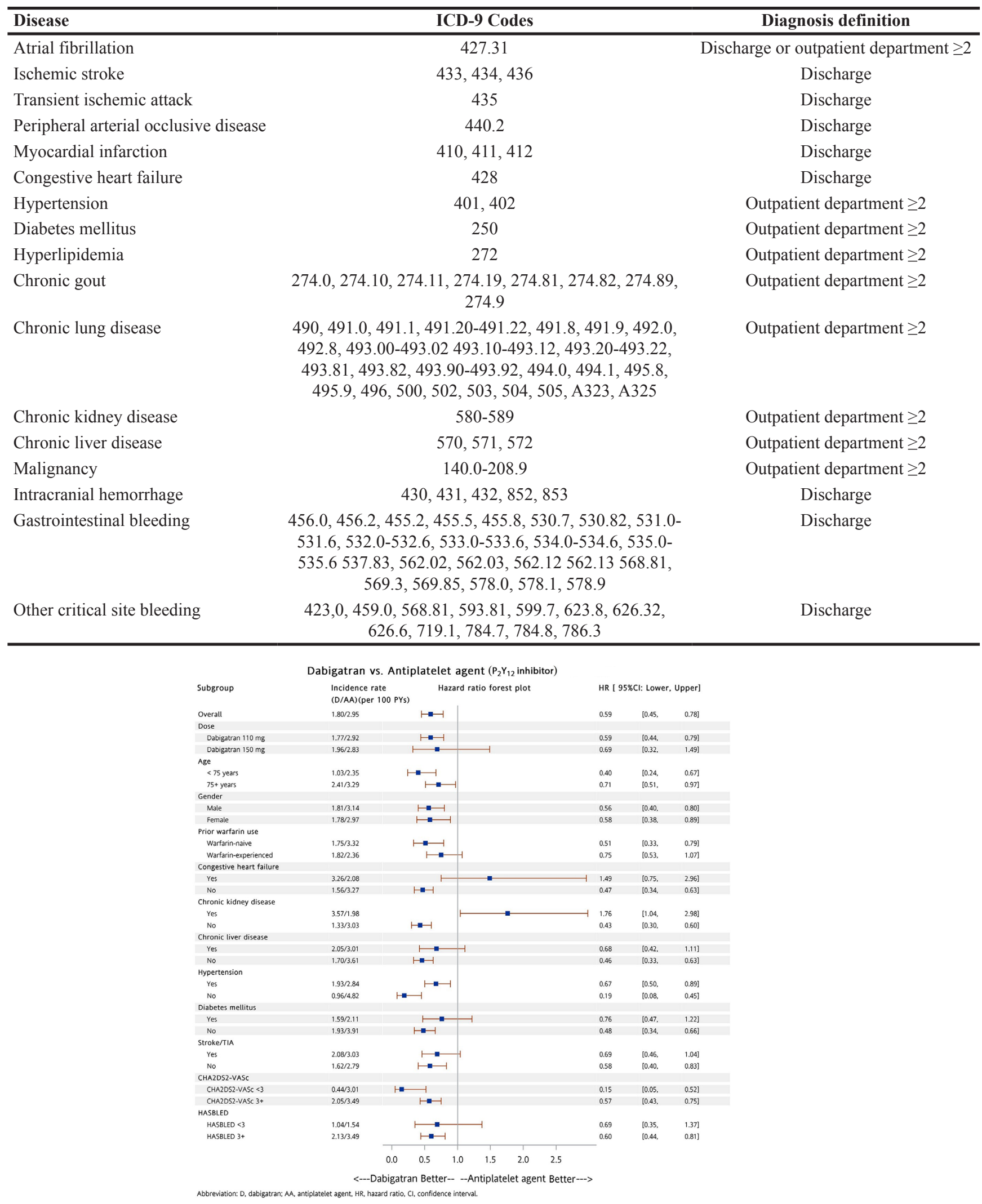

Figure 4: Forest plot of hazard ratio of all major bleeding for NVAF patients taking dabigatran versus $P_{2} Y_{12}$ inhibitor after propensity score weighting. Dabigatran was associated with a lower risk of all major bleeding events compared with $\mathrm{P}_{2} \mathrm{Y}_{12}$ inhibitor in most subgroups. The abbreviations as in Figure 1. 
thrombotic therapy with an enrollment of 6,600 and 8,238 Asians taking dabigatran and AA respectively. Our data indicated that dabigatran was significantly associated with a lower risk of all major bleeding compared with AA during identical follow-up periods. The RELY subgroup analysis previously indicated that the major bleeding risk was significantly lower among Asians on either standard or low dose dabigatran compared to warfarin. [24] In contrast, the ROCKET-AF trial and recent real world data showed that rivaroxaban, which was in contrast to other
NOACs (apixaban, dabigatran, and edoxaban), has a similar risk of major bleeding comparable with warfarin. [25-30] The recent real world data further demonstrated that rivaroxaban caused more $\mathrm{ICH}, \mathrm{GIB}$ and all major bleeding than dabigatran. [31] It was therefore expected that rivaroxaban may be associated with a comparable risk of major bleeding as AA. However, our present data showed that in real world practice in Asia, both dabigatran and rivaroxaban were associated with a lower risk of ICH compared to AA.

Rivaroxaban vs. Antiplatelet agent (Aspirin)

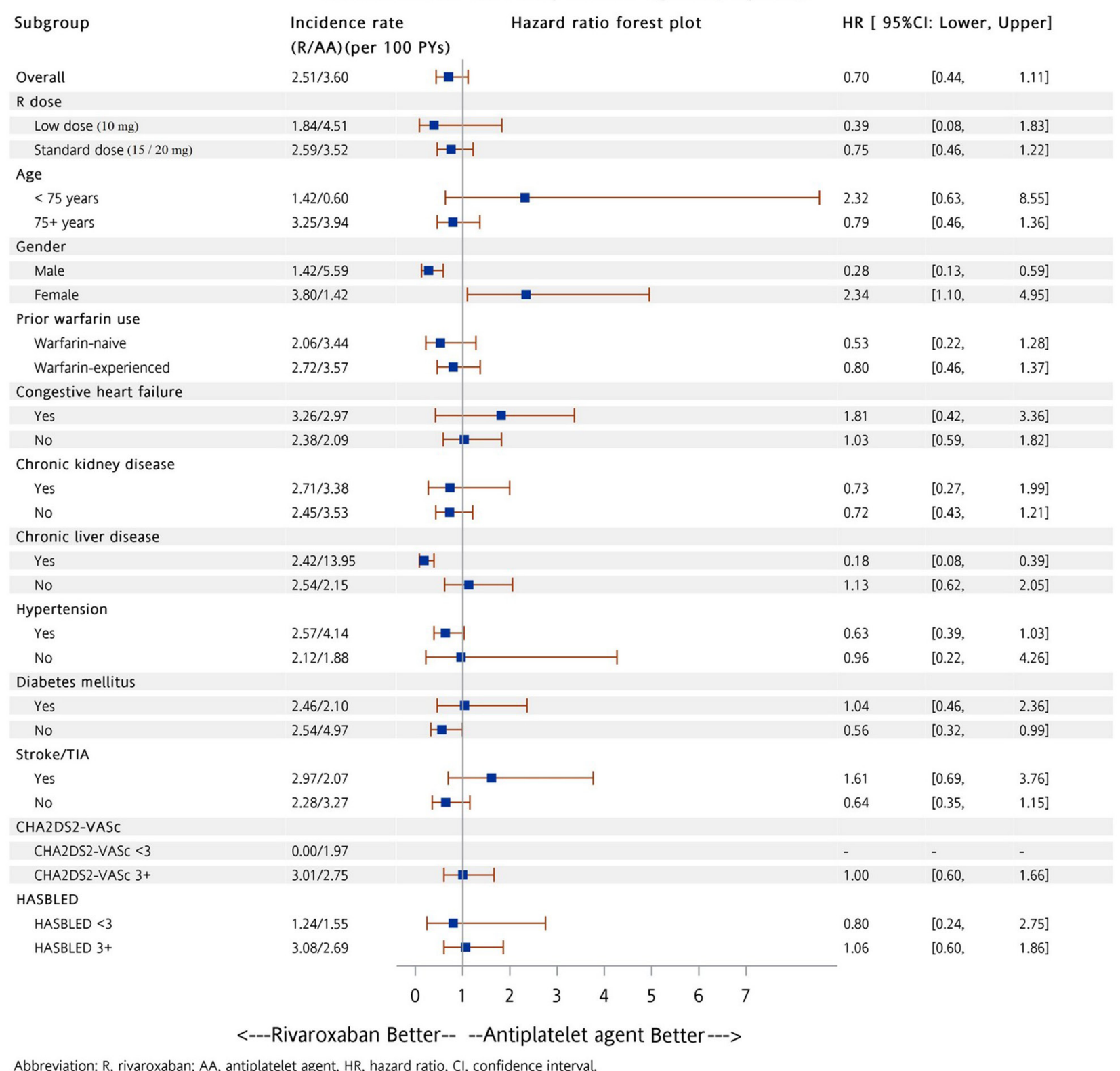

Figure 5: Forest plot of hazard ratio of all major bleeding for NVAF patients taking rivaroxaban versus aspirin after propensity score weighting. Rivaroxaban was associated with a similar risk of all major bleeding as compared with aspirin users in most subgroups. The abbreviations as in Figure 1. 


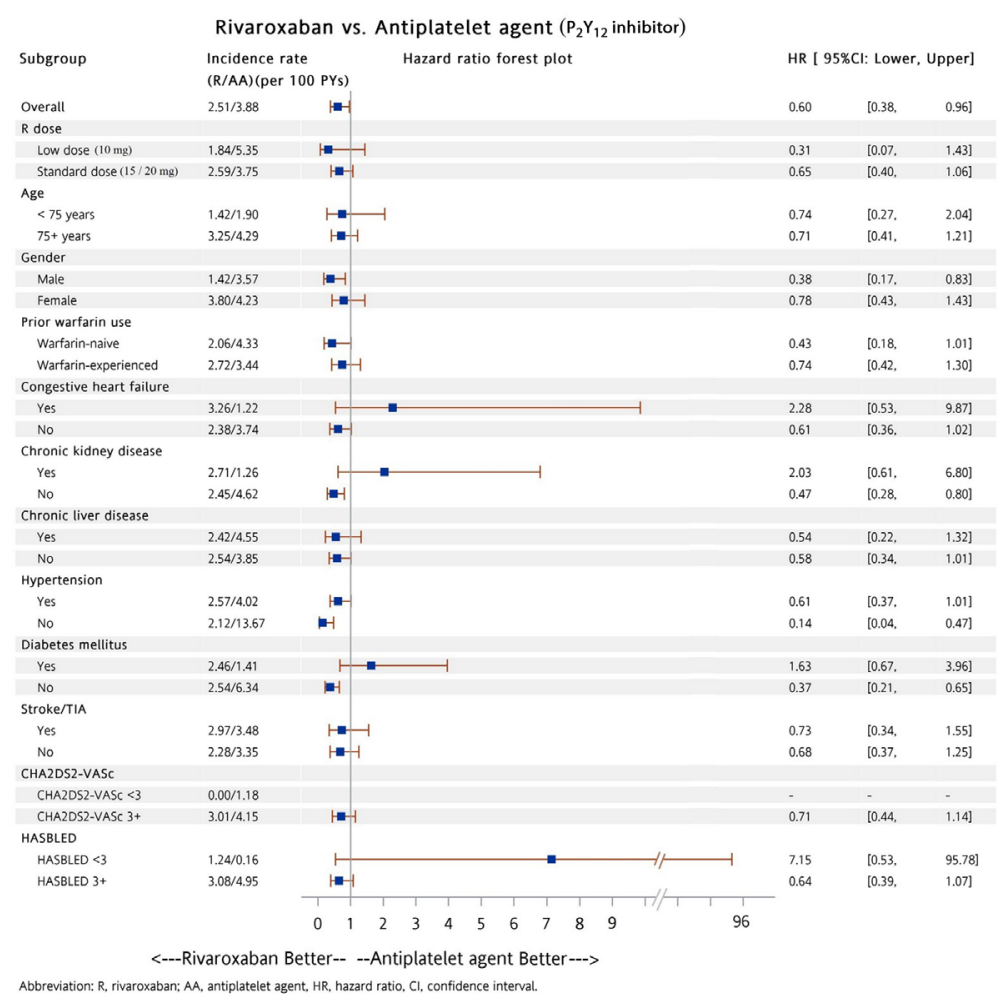

Figure 6: Forest plot of hazard ratios of all major bleeding for NVAF patients taking rivaroxaban versus $P_{2} Y_{12}$ inhibitor after propensity score weighting. Warfarin was associated with a similar risk of all major bleeding as compared with $\mathrm{P}_{2} \mathrm{Y}_{12}$ inhibitor in most subgroups. The abbreviations as in Figure 1.

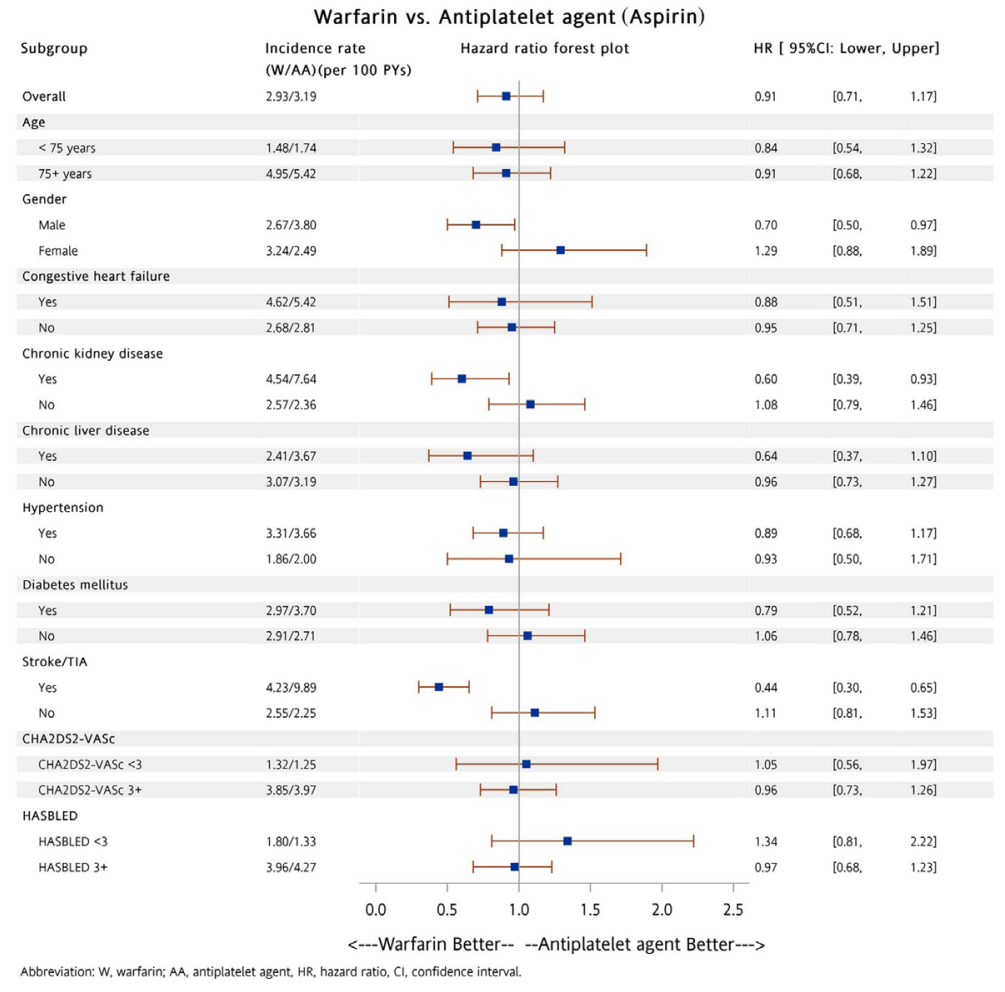

Figure 7: Forest plot of hazard ratios of all major bleeding for NVAF patients taking warfarin versus aspirin after propensity score weighting. Warfarin was associated with a similar risk of all major bleeding as compared with aspirin in most subgroups. The abbreviations as in Figure 1. 


\section{Study limitations}

First, we cannot evaluate several laboratory parameters including hemoglobin, labile INR, renal and liver function test, and other potential confounders from the national database. Second, although we included an extensive number of variables in our model and achieved a close balance for most factors, residual confounding by unmeasured factors cannot be excluded. Third, this study had a relative short follow-up period. Fourth, the data regarding apixaban and edoxaban were not available. Finally, because dabigatran largely depends on renal excretion, it is possible that the physicians could avoid the use of dabigatran for patients with a further impaired renal function. Consequently, the baseline renal function may be better for the dabigatran group compared to

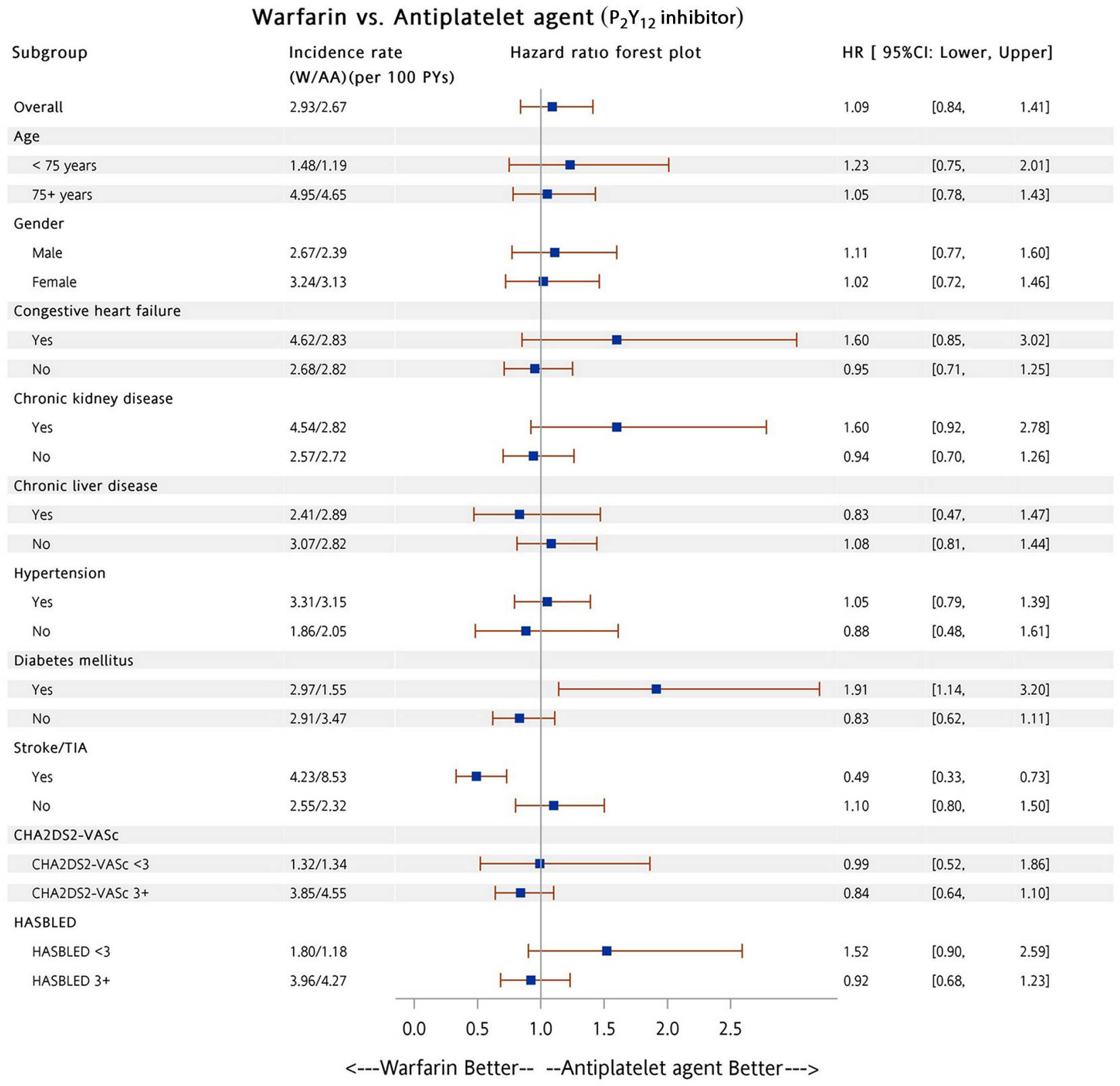

Abbreviation: W, warfarin; $\mathrm{AA}$, antiplatelet agent, $\mathrm{HR}$, hazard ratio, $\mathrm{Cl}$, confidence interval.

Figure 8: Forest plot of hazard ratios of all major bleeding for NVAF patients taking warfarin versus $P_{2} Y_{12}$ inhibitor after propensity score weighting. Warfarin was associated with a similar risk of all major bleeding as compared with $\mathrm{P}_{2} \mathrm{Y}_{12}$ inhibitor in most subgroups. The abbreviations as in Figure 1. 


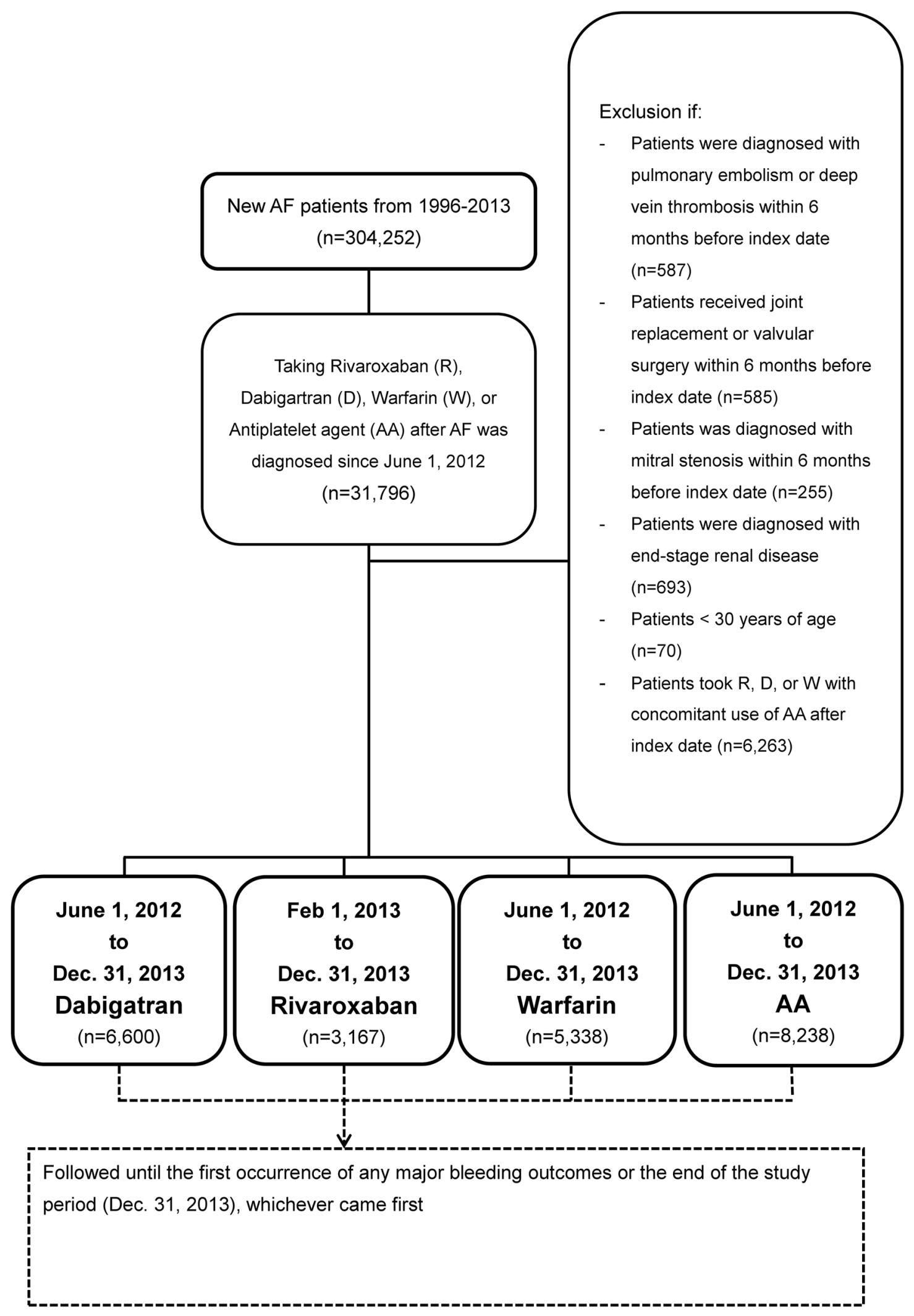

Figure 9: Enrollment of patients with NVAF. A total of 304,252 new non-valvular atrial fibrillation (NVAF) patients including 6,600 dabigatran (D), 3,167 rivaroxaban (R), 5,338 warfarin (W), and 8,238 antiplatelet agent (AA) users were enrolled in this study from June 1, 2012 to December 31, 2013. Rivaroxaban was enrolled in this study from February 1, 2013 duo to its approval at that day in Taiwan. 
other group, which may explain for the better safety profiles with dabigatran compared to other study group. Because the NHIRD does not have important laboratory data including serum creatinine level, we cannot clarify the issue if the favorable outcome of safety issue for dabigatran was contributed from the better baseline renal function or not.

\section{MATERIALS AND METHODS}

\section{Study population}

The Taiwan National Health Insurance (NHI) system is a mandatory universal health insurance program providing comprehensive medical care coverage to all Taiwanese residents. The NHI research database (NHIRD) of the National Health Research Institutes of Taiwan included detailed health care information for more than $99 \%$ of the Taiwanese population (23 million enrollees) in 2014. [8] The study was approved by the Institutional Review Board of Chang-Gung Medical Foundation.

This study evaluated a national cohort with four study groups (dabigatran, rivaroxaban, warfarin and AA). Figure 9 showed the flowchart of the study cohort. A total of 304,252 new AF patients were identified between January, 1996 and December, 2013. AF was diagnosed based on International Classification of Diseases 9th, Clinical Modification (ICD-9-CM) codes (427.31), in either in-patient once or outpatient department twice. We identified patients with a first time prescription of NOACs dabigatran (from June 1, 2012) and rivaroxaban (from February 1, 2013), as well as patients who started warfarin or AA treatment (from June 1, 2012) up to December 31, 2013 after an established diagnosis of AF. [9, 32] Index date for each study group was defined as the first date when medication was prescribed. The excluded patients were presented in Figure 9. Patients taking NOACs or warfarin with concomitant use of AA after the index date $(n=6,263)$ were also excluded. The follow-up period was from the index date until the occurrence of the first study outcomes or the end of the study period (December 31, 2013), whichever came first.

\section{Study outcomes}

The three study outcomes defined to determine the safety profiles for dabigatran, rivaroxaban, warfarin and AA were 1) $\mathrm{ICH}, 2$ ) GIB, and 3) all major bleeding events. The study outcomes had to be a discharge diagnosis. Major bleeding was defined a hospitalized bleeding event with hemorrhage into a critical site (e.g., gastrointestinal, intracranial, intraspinal, intraarticular, intraocular, pericardial, retroperitoneal, or intramuscular with compartment syndrome). ICH was defined with the use of codes for atraumatic hemorrhage. [9] The ICD-9$\mathrm{CM}$ codes used in the study outcomes are summarized in Table 8. It notes that the same patient could have more than one study outcomes and could have the same study outcomes several times during the study duration. However, only the study outcomes which appeared first were counted, because patients were managed differently based on their study outcomes.

\section{Covariates}

Information for risk factors of cardiovascular events and bleeding, and use of medication at baseline was obtained from claim records with the above diagnoses or medication codes prior to the index date. History of bleeding was confined to events within 6 months preceding the index date. A history of specific prescribed medicines was confined to medications used at least once within 3 months preceding the index date. The $\mathrm{CHA}_{2} \mathrm{DS}_{2}-$ VASc score predicted the risk of ischemic stroke in AF patients, and the HAS-BLED score predicted the risk of bleeding in AF patients treated with oral anticoagulant or antiplatelet. $[10,11]$

\section{Statistical analysis}

The effects of dabigatran, rivaroxaban and warfarin on study outcomes were estimated using the propensity score method. Inverse probability of treatment weights (IPTW) of propensity scores was used to balance covariates across the two study groups. [12] The balance of potential confounders at baseline (index date) between the two study groups was assessed using absolute standardized mean difference (ASMD), rather than using statistical testing, because balance is a property of the sample and not of an underlying population. The value of ASMD $\leq 0.1$ indicates a negligible difference in potential confounders between the two study groups. Risk of study outcomes over time for dabigatran, rivaroxaban or warfarin compared with AA (reference) was obtained using survival analysis (Kaplan-Meier method for univariate analysis and Cox proportional hazards regression for multivariate analysis) after IPTW. Statistical significance was defined as a $P$ value of $<0.05$. All statistical analyses were performed using SAS 9.3 (SAS Institute Inc., Cary, North Carolina).

\section{CONCLUSIONS}

Dabigatran use was associated with significantly lower risk of all major bleeding events compared to AA in a large Asian cohort with NVAF. In contrast, patients in rivaroxaban groups had a similar risk of major bleeding compared to the AA group. The findings implicated that 
dabigatran may be a safer alternative to aspirin for Asian AF patients.

\section{Abbreviations}

AF, atrial fibrillation

GIB, gastrointestinal bleeding

ICH, intracranial hemorrhage

INR, international normalized ratio

NOAC, non-vitamin $\mathrm{K}$ antagonist oral anticoagulant

NVAF, non-valvular atrial fibrillation

OAC, oral anticoagulant

VKA, vitamin K Antagonists

NHIRD, National Health Insurance Research Database

TTR, time in therapeutic range

\section{CONFLICTS OF INTEREST}

None.

\section{GRANT SUPPORT}

This study was supported by grants 1022628-B-182 -011 -MY3, 102-2314-B-182A-053 -MY3 from the Ministry of Science and Technology and CMRPG3B0991-3, CMRPG3F0991-3, CMRPG3D1371-3, CMRPD1F0252 and BMRP300 from the Chang-Gung Memorial Hospital, Linkou, Taiwan.

\section{REFERENCES}

1. Hart RG, Pearce LA, Aguilar MI. Meta-analysis: antithrombotic therapy to prevent stroke in patients who have nonvalvular atrial fibrillation. Ann Intern Med. 2007; 146:857-867.

2. Fang MC, Stafford RS, Ruskin JN, Singer DE. National trends in antiarrhythmic and antithrombotic medication use in atrial fibrillation. Arch Intern Med. 2004;164:55-60.

3. DeWilde S, Carey IM, Emmas C, Richards N, Cook DG. Trends in the prevalence of diagnosed atrial fibrillation, its treatment with anticoagulation and predictors of such treatment in UK primary care. Heart. 2006;92:1064-1070.

4. Shen AY, Yao JF, Brar SS, Jorgensen MB, Chen W. Racial/ ethnic differences in the risk of intracranial hemorrhage among patients with atrial fibrillation. J Am Coll Cardiol. 2007;50:309-315.

5. van Asch CJ, Luitse MJ, Rinkel GJ, van der Tweel I, Algra A, Klijn CJ. Incidence, case fatality, and functional outcome of intracerebral haemorrhage over time, according to age, sex, and ethnic origin: a systematic review and metaanalysis. Lancet Neurol. 2010;9:167-176.

6. Oh S, Goto S, Accetta G, Angchaisuksiri P, Camm AJ, Cools F, Haas S, Kayani G, Koretsune Y, Lim TW, Misselwitz F, van Eickels M, Kakkar AK; GARFIELD-AF
Investigators. Vitamin $\mathrm{K}$ antagonist control in patients with atrial fibrillation in Asia compared with other regions of the world: real-world data from the GARFIELD-AF registry. Int J Cardiol. 2016;223:543-547.

7. Connolly SJ, Eikelboom J, Joyner C, Diener HC, Hart R, Golitsyn S, Flaker G, Avezum A, Hohnloser SH, Diaz R, Talajic M, Zhu J, Pais P, et al. Apixaban in patients with atrial fibrillation. N Engl J Med. 2011;364:806-817.

8. National Health Insurance Administration, Ministry of Health \& Welfare, Taiwan. The National Health Insurance Statistics, 2014. at http://www.nhi.gov.tw/English/webdata/webdata. aspx?menu=11\&menu_id=296\&WD_ID=296\&webdata $\mathrm{id}=4835$.

9. Chan YH, Kuo CT, Yeh YH, Chang SH, Wu LS, Lee HF, Tu HT, See LC. Thromboembolic, bleeding, and mortality risks of rivaroxaban and dabigatran in Asians with nonvalvular atrial fibrillation. J Am Coll Cardiol. 2016;68:1389-1401.

10. Pamukcu B, Lip GY, Lane DA. Simplifying stroke risk stratification in atrial fibrillation patients: implications of the CHA2DS2-VASc risk stratification scores. Age Ageing. 2010;39:533-535.

11. Pisters R, Lane DA, Nieuwlaat R, de Vos CB, Crijns HJ, Lip GY. A novel user-friendly score (HAS-BLED) to assess 1-year risk of major bleeding in patients with atrial fibrillation: the Euro Heart Survey. Chest. 2010;138:1093-1100.

12. Austin PC. The use of propensity score methods with survival or time-to-event outcomes: reporting measures of effect similar to those used in randomized experiments. Stat Med. 2014;33:1242-1258.

13. Mant J, Hobbs FD, Fletcher K, Roalfe A, Fitzmaurice D, Lip GY, Murray E; BAFTA investigators; Midland Research Practices Network (MidReC). Warfarin versus aspirin for stroke prevention in an elderly community population with atrial fibrillation (the Birmingham Atrial Fibrillation Treatment of the Aged Study, BAFTA): a randomised controlled trial. Lancet. 2007;370:493-503.

14. Sato H, Ishikawa K, Kitabatake A, Ogawa S, Maruyama Y, Yokota Y, Fukuyama T, Doi Y, Mochizuki S, Izumi T, Takekoshi N, Yoshida K, Hiramori K, et al. Low-dose aspirin for prevention of stroke in low-risk patients with atrial fibrillation: Japan Atrial Fibrillation Stroke Trial. Stroke. 2006;37:447-451.

15. Siu CW, Lip GY, Lam KF, Tse HF. Risk of stroke and intracranial hemorrhage in 9727 Chinese with atrial fibrillation in Hong Kong. Heart Rhythm. 2014;11:1401-1408.

16. Huisman MV, Rothman KJ, Paquette M, Teutsch C, Diener HC, Dubner SJ, Halperin JL, Ma C, Zint K, Elsaesser A, Bartels DB, Lip GY; GLORIA-AF Investigators. Antithrombotic treatment patterns in patients with newly diagnosed nonvalvular atrial fibrillation: the GLORIA-AF registry, phase II. Am J Med. 2015;128:1306-1313 e1301.

17. Ho CW, Ho MH, Chan PH, Hai JJ, Cheung E, Yeung CY, Lau KK, Chan KH, Lau CP, Lip GY, Leung GK, Tse HF, 
Siu CW. Ischemic stroke and intracranial hemorrhage with aspirin, dabigatran, and warfarin: impact of quality of anticoagulation control. Stroke. 2015;46:23-30.

18. Nieuwlaat R, Connolly BJ, Hubers LM, Cuddy SM, Eikelboom JW, Yusuf S, Connolly SJ; ACTIVE Investigators. Quality of individual INR control and the risk of stroke and bleeding events in atrial fibrillation patients: a nested case control analysis of the ACTIVE W study. Thromb Res. 2012;129:715-719.

19. Kirchhof P, Benussi S, Kotecha D, Ahlsson A, Atar D, Casadei B, Castella M, Diener HC, Heidbuchel H, Hendriks J, Hindricks G, Manolis AS, Oldgren J. 2016 ESC Guidelines for the management of atrial fibrillation developed in collaboration with EACTS. Eur Heart J. 2016;37:2893-2962.

20. Ambrosi P, Daumas A, Villani P, Giorgi R. Metaanalysis of major bleeding events on aspirin versus vitamin $\mathrm{K}$ antagonists in randomized trials. Int J Cardiol. 2017;230:572-576.

21. Wang KL, Lip GY, Lin SJ, Chiang CE. Non-vitamin K antagonist oral anticoagulants for stroke prevention in Asian patients with nonvalvular atrial fibrillation: meta-analysis. Stroke. 2015;46:2555-2561.

22. Chiang CE, Wu TJ, Ueng KC, Chao TF, Chang KC, Wang CC, Lin YJ, Yin WH, Kuo JY, Lin WS, Tsai CT, Liu YB, Lee KT. 2016 Guidelines of the Taiwan heart rhythm society and the Taiwan society of cardiology for the management of atrial fibrillation. J Formos Med Assoc. 2016;115:893-952.

23. Chan PH, Li WH, Hai JJ, Chan KH, Tse HF, Cheung BM, Chan EW, Wong IC, Leung WK, Hung IF, Lip GY, Siu CW. Gastrointestinal haemorrhage in atrial fibrillation patients: impact of quality of anticoagulation control. Eur Heart $\mathbf{J}$ Cardiovasc Pharmacother. 2015;1:265-272.

24. Hori M, Connolly SJ, Zhu J, Liu LS, Lau CP, Pais P, Xavier D, Kim SS, Omar R, Dans AL, Tan RS, Chen JH, Tanomsup $\mathrm{S}$, et al. Dabigatran versus warfarin: effects on ischemic and hemorrhagic strokes and bleeding in Asians and non-Asians with atrial fibrillation. Stroke. 2013;44:1891-1896.

25. Connolly SJ, Ezekowitz MD, Yusuf S, Eikelboom J, Oldgren J, Parekh A, Pogue J, Reilly PA, Themeles E, Varrone J, Wang S, Alings M, Xavier D, et al. Dabigatran versus warfarin in patients with atrial fibrillation. N Engl $\mathrm{J}$ Med. 2009;361:1139-1151.

26. Patel MR, Mahaffey KW, Garg J, Pan G, Singer DE, Hacke W, Breithardt G, Halperin JL, Hankey GJ, Piccini JP, Becker RC, Nessel CC, Paolini JF, et al. Rivaroxaban versus warfarin in nonvalvular atrial fibrillation. $\mathrm{N}$ Engl $\mathrm{J}$ Med. 2011;365:883-891.

27. Granger CB, Alexander JH, McMurray JJ, Lopes RD, Hylek EM, Hanna M, Al-Khalidi HR, Ansell J, Atar D, Avezum A, Bahit MC, Diaz R, Easton JD, et al. Apixaban versus warfarin in patients with atrial fibrillation. $\mathrm{N}$ Engl $\mathrm{J}$ Med. 2011;365:981-992.

28. Giugliano RP, Ruff CT, Braunwald E, Murphy SA, Wiviott SD, Halperin JL, Waldo AL, Ezekowitz MD, Weitz JI, Špinar J, Ruzyllo W, Ruda M, Koretsune Y, et al. Edoxaban versus warfarin in patients with atrial fibrillation. N Engl J Med. 2013;369:2093-2104.

29. Larsen TB, Skjoth F, Nielsen PB, Kjaeldgaard JN, Lip GY. Comparative effectiveness and safety of non-vitamin $\mathrm{K}$ antagonist oral anticoagulants and warfarin in patients with atrial fibrillation: propensity weighted nationwide cohort study. BMJ. 2016;353:i3189.

30. Yao X, Abraham NS, Sangaralingham LR, Bellolio MF, McBane RD, Shah ND, Noseworthy PA. Effectiveness and safety of dabigatran, rivaroxaban, and apixaban versus warfarin in nonvalvular atrial fibrillation. J Am Heart Assoc. 2016.

31. Graham DJ, Reichman ME, Wernecke M, Hsueh YH, Izem R, Southworth MR, Wei Y, Liao J, Goulding MR, Mott K, Chillarige Y, MaCurdy TE, Worrall C, et al. Stroke, bleeding, and mortality risks in elderly medicare beneficiaries treated with dabigatran or rivaroxaban for nonvalvular atrial fibrillation. JAMA Intern Med. 2016;176:1662-1671.

32. Chan YH, Wu LS, Chang SH, Lee HF, Liu JR, See LC, Yeh YH, Kuo CT. Young male patients with atrial fibrillation and CHA2DS2-VASc Score of 1 may not need anticoagulants: a nationwide population-based study. PLoS One. 2016;11:e0151485. 